\title{
Temporal Expression of Key Angioregulatory Proteins in Response to Exercise and Detraining
}

\author{
Sara A. Olenich \\ West Virginia University
}

Follow this and additional works at: https://researchrepository.wvu.edu/etd

\section{Recommended Citation}

Olenich, Sara A., "Temporal Expression of Key Angioregulatory Proteins in Response to Exercise and Detraining" (2013). Graduate Theses, Dissertations, and Problem Reports. 4986.

https://researchrepository.wvu.edu/etd/4986

This Thesis is protected by copyright and/or related rights. It has been brought to you by the The Research Repository @ WVU with permission from the rights-holder(s). You are free to use this Thesis in any way that is permitted by the copyright and related rights legislation that applies to your use. For other uses you must obtain permission from the rights-holder(s) directly, unless additional rights are indicated by a Creative Commons license in the record and/ or on the work itself. This Thesis has been accepted for inclusion in WVU Graduate Theses, Dissertations, and Problem Reports collection by an authorized administrator of The Research Repository @ WVU. For more information, please contact researchrepository@mail.wvu.edu. 


\title{
Temporal Expression of Key Angioregulatory Proteins in Response to Exercise and Detraining
}

\author{
Sara A. Olenich \\ Thesis submitted to the College of Medicine \\ at West Virginia University \\ In partial fulfillment of the requirements \\ for the degree of \\ Master of Science \\ in \\ Exercise Physiology
}

\section{Mark Olfert Ph.D., Committee Chair \\ Stephen Alway, Ph.D. \\ Emidio Pistilli, Ph.D.}

School of Medicine

Morgantown, West Virginia

2013

Keywords: Training, Detraing, VEGF, Thrombospondin-1, Nucleolin, Endostatin, MMP-2, MMP-9, Angiogenesis 


\section{Abstract \\ Temporal Expression of Key Angioregulatory Proteins in Response to Exercise and Detraining}

\section{Sara A. Olenich}

Angiogenesis is an important adaptation to exercise, occurring in response to a multitude of different stimuli including: shear stress, mechanical stretch, ischemia, electrical stimulation, and exercise. Current thinking suggests skeletal muscle angiogenesis is a temporal process controlled by a balance between positive and negative angiogenic proteins. But there is limited information on what molecular mediators control skeletal muscle angiogenesis in this time line, creating a critical need to clarify how individual protein responses regulate physiologic skeletal muscle angiogenesis in response to exercise training and/or physical deconditioning. Our objective is to characterize the temporal expression of several key positive (VEGF, MMP-2, MMP-9, nucleolin) and negative (TSP-1, endostatin) angiogenic factors under basal conditions, after acute exercise, in response to training, and after detraining. The central hypothesis is that training and deconditioning will cause temporally coordinated changes in positive and negative angiogenic regulators in response to exercise training, which will be reversed during detraining. 


\section{TABLE OF CONTENT}

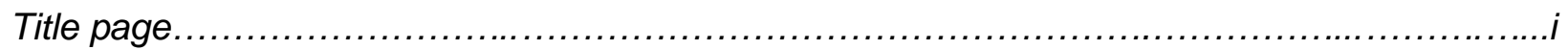

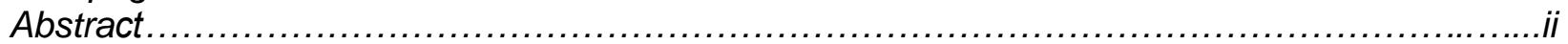

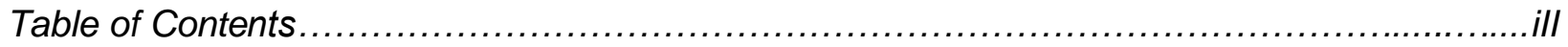

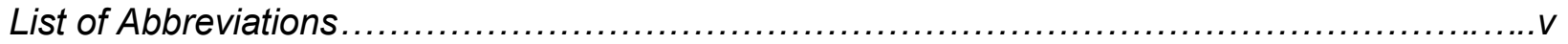

TABLE OF CONTENTS:

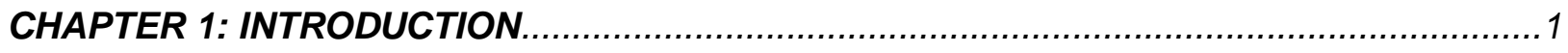

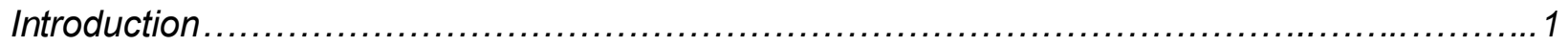

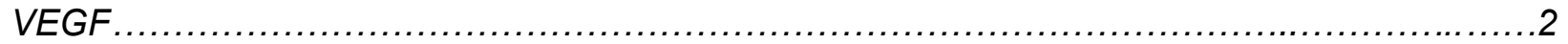

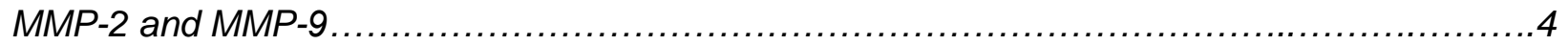

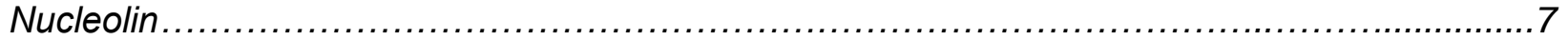

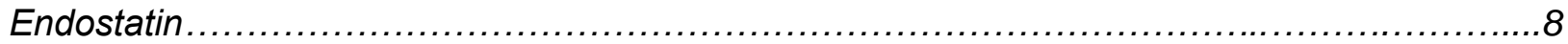

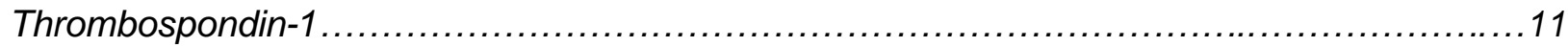

Integrated angiogenic protein response to exercise ........................................... 12

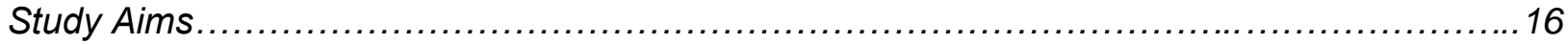

\section{CHAPTER 2: TEMPORAL RESPONSE OF POSITIVE AND NEGATIVE REGULATORS IN RESPONSE TO ACUTE AND CHRONIC EXERCISE}

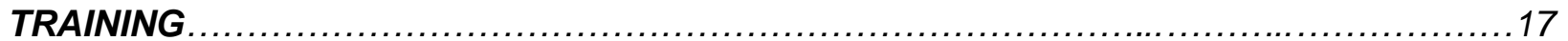

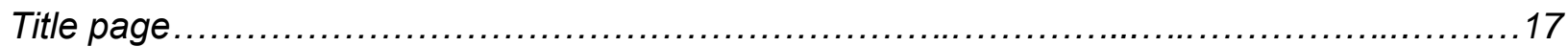

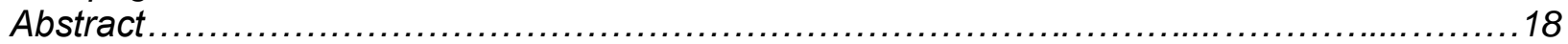

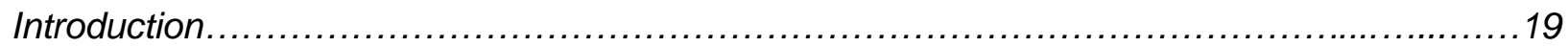

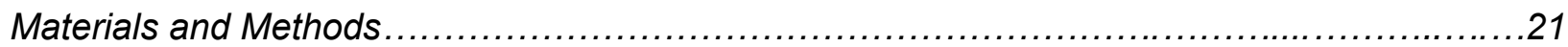

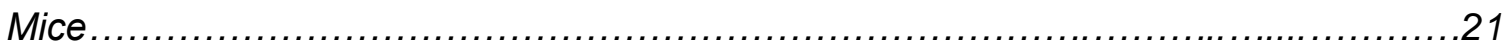

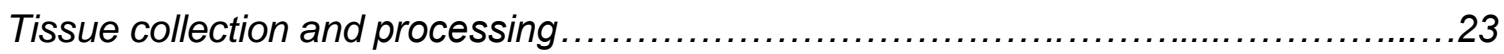

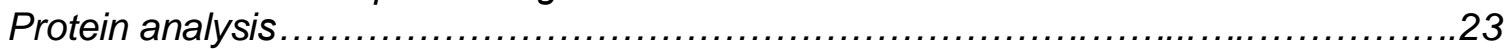

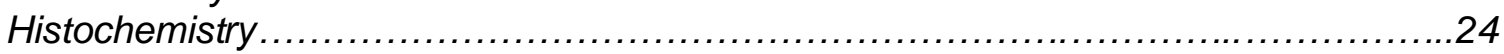

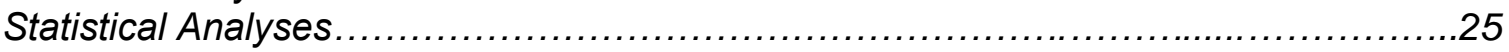

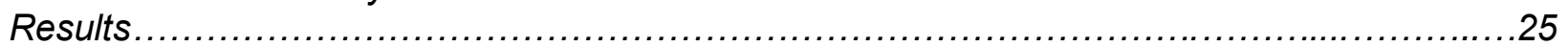

Study 1: Time course response to single bout of acute exercise .........................25

Study 2: Temporal response of angiogenic regulators to exercise training.................25

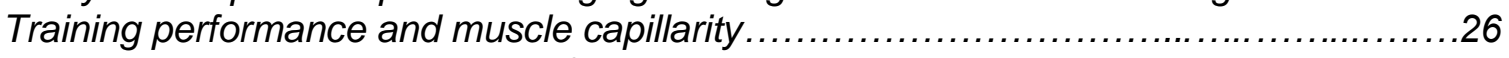

Basal muscle protein expression following training ......................................26

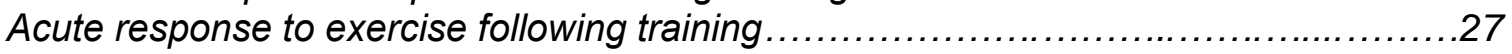

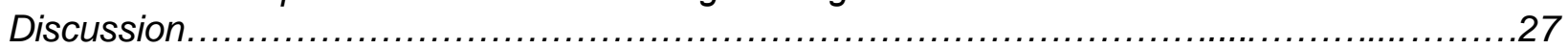

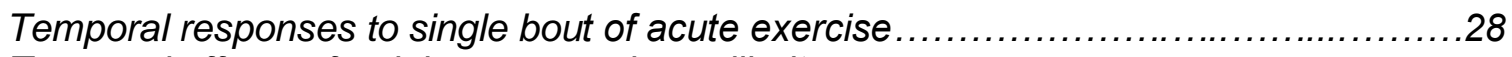

Temporal effects of training on muscle capillarity ......................................... 31

Basal response of angiogenic regulators following exercise training ......................32

Acute exercise responses of angiogenic regulators following exercise training.............36

Conclusion 38

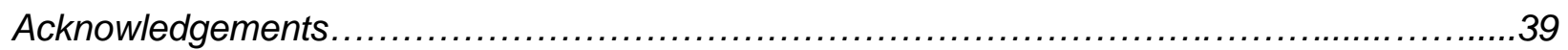

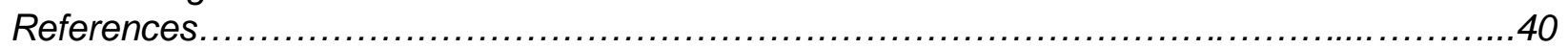

Tables and

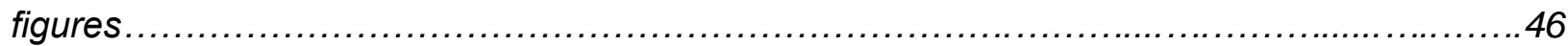

Table 1: Acute exercise time course age, body and muscle mass.......................46

Table 2: Training age, body and muscle mass.........................................47

Table 3 Running wheel training data.................................................48

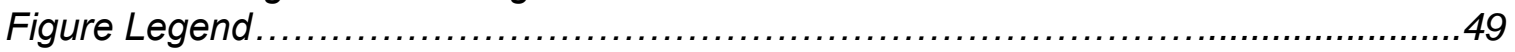

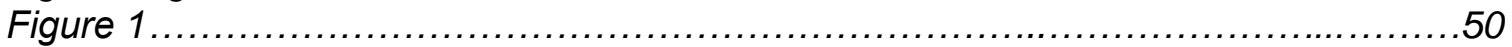




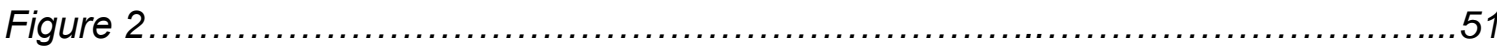

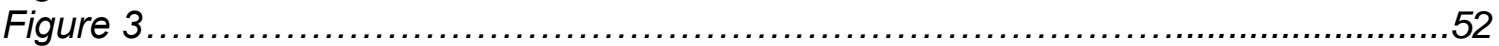

CHAPTER 3: EFFECTS OF DETRAINING ON THE TEMPORAL EXPRESSION OF KEY POSITIVE AND NEGATIVE ANGIOREGULATORY PROTEINS IN SKELETAL MUSCLE....53

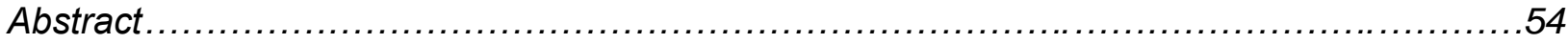

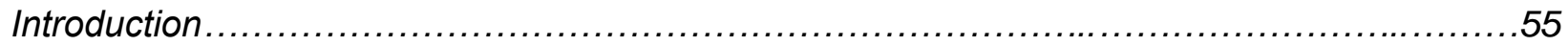

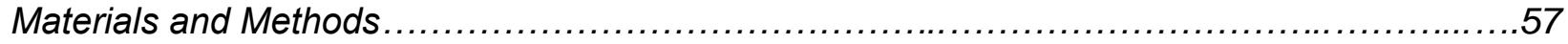

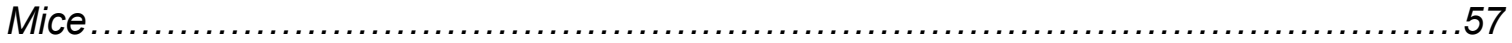

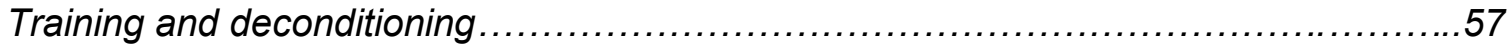

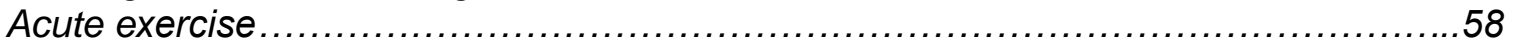

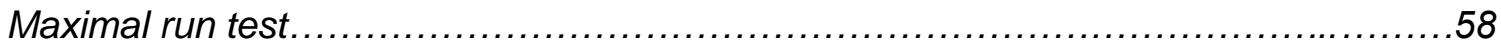

Tissue collection and protein expression........................................... 59

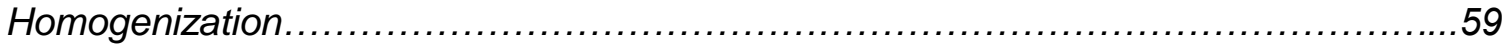

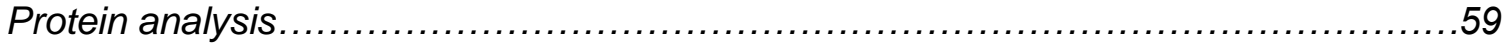

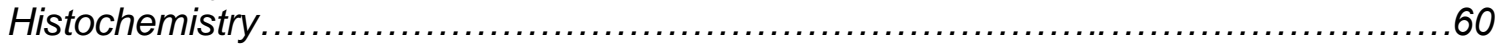

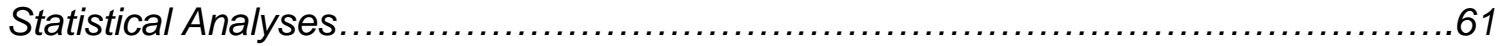

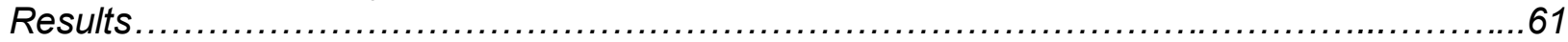

Animal Body Mass and Muscle Characteristics....................................61

Training Behavior and Aerobic Performance.......................................62

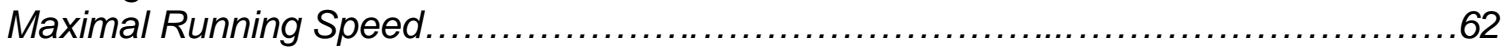

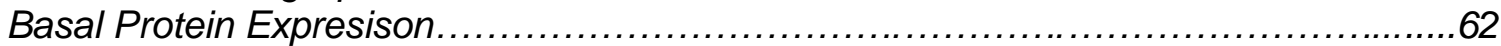

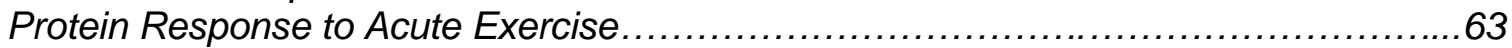

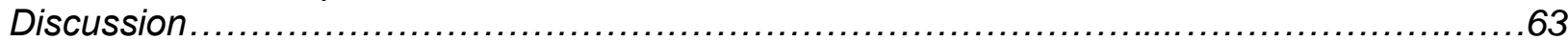

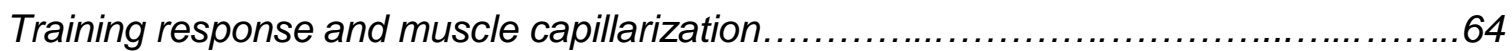

Detraining and protein expression in skeletal muscles..................................6

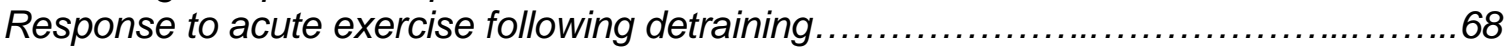

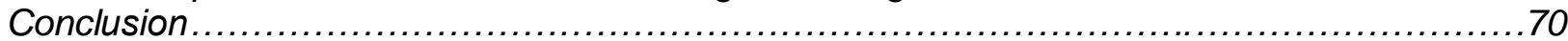

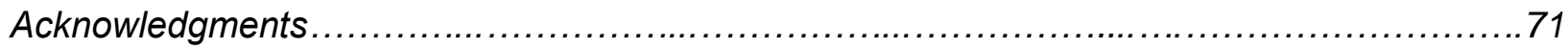

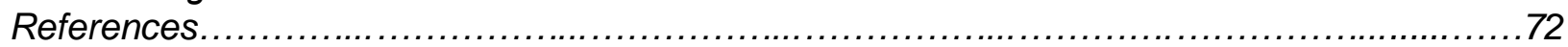

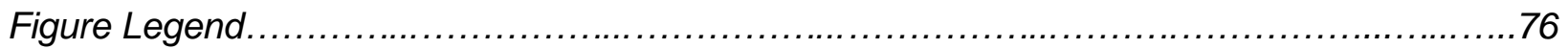

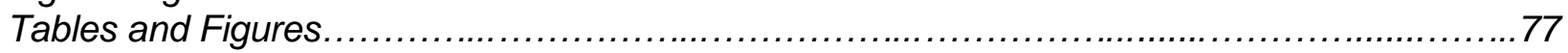

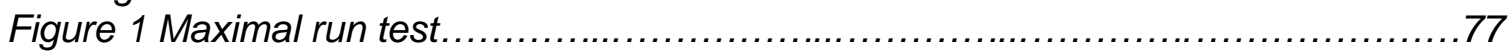

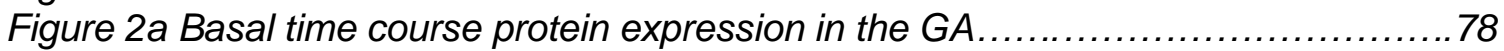

Figure $2 b$ Basal time course protein expression in the $P L T \ldots \ldots \ldots \ldots \ldots \ldots \ldots \ldots \ldots \ldots . \ldots \ldots$

Figure $2 c$ Basal time course protein expression in the SOL............................. 80

Figure 3 Acute time course response to exercise................................... 81

Table 1. Wheel Running characteristics, animal characteristics and C:F ratio...........82

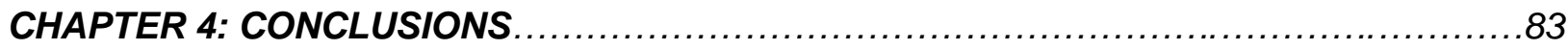

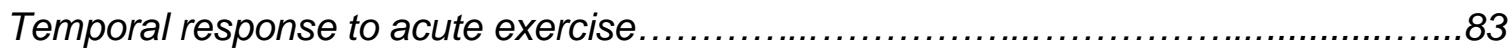

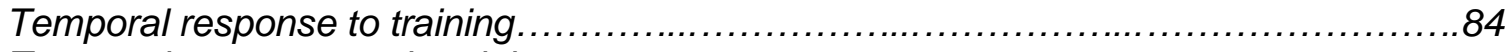

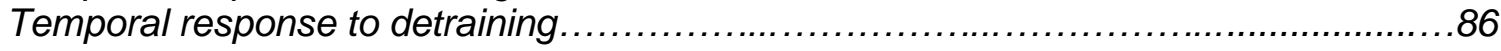




\section{List of Symbols, Abbreviations, or Nomenclature}

\section{Terms:}

C:F ratio Capillary to Fiber ratio

ECM Extra Cellular Matrix

Flt-1 Fetal Liver Kinase-1

GA Gastrocnemius

KDR Kinase Domain-Containing Receptor

KO Knock Out

MMP Matrix Metalloproteinase

PLT Plantaris

SOL Soleus

TIMP Tissue Inhibitor of Metalloproteinases

TSP-1 Thrombospondin-1

VEGF Vascular Endothelial Growth Factor

VEGFR2 VEGF Receptor 2 


\section{CHAPTER 1: INTRODUCTION}

Increasing skeletal muscle vascularity in active muscle is recognized to be important in improving the surface area on which substrate/metabolism/oxygen exchange can take place in order to meet the high metabolic demands (of skeletal muscle) imposed during periods of increased physical activity (Snyder 1987, Wagner, Roca et al. 1990, Snyder, Farrelly et al. 1992, Wagner 1996, Mathieu-Costello and Hepple 2002, Howlett, Kirkton et al. 2008). Under normal conditions endothelial cells, the cells which line blood vessels, have a slow turnover rate of about a thousand days, but when called upon, such as in wound healing, endothelial cells can turnover in five days (Folkman and Shing 1992). Likewise, exercise can induce significant changes of skeletal muscle capillarity in 7 days (mouse) (Waters, Rotevatn et al. 2004) or as little as 2-4 days (rabbit) when electrically stimulated for 8 hours a day (Brown, Cotter et al. 1976). The growth of vasculature occurs relatively quickly, which is important to meet the increased demand of skeletal muscle. Yet, more fascinating than the speed of angiogenesis is the temporal coordination and organization of numerous pro and anti angiogenic mitogens in order for sprouting vessels to form without overt vascular disruption.

Understanding the temporal expression patterns of essential angiogenic peptides, such as vascular endothelial growth factor (VEGF), matrix metalloproteinase (MMP) -2, MMP-9, nucleolin, endostatin, and thrombospondin-1 (TSP-1) in response to angiogenic-inducing stimuli (e.g. exercise) is needed to better understand the physiologic regulation of angiogenesis. For example, VEGF stimulates vascular permeability allowing for extravasation of plasma proteins, endothelial cell proliferation, migration, and adhesion for vessel lumen formation. Nucleolin may help mediate VEGF to the nucleus while presence of MMP-2 and MMP-9 are needed to disrupt the extracellular matrix surrounding the existing vessel in order to accommodate the new vascular outcropping (Huang, Shi et al. 2006). Plasma proteins, including fibrinogen and plasminogen, escape the blood stream through permeable vessels and are capable of initiating a basic scaffold on which endothelial cells begin can migrate and to form a cord or tube-like 
structure. After lumen formation the vessels are still unstable until mural cells migrate in and differentiate into pericytes or smooth muscle cells. As patent vessels are formed, withdrawal and/or inhibition of the pro-angiogenic signals are needed for maturation and stabilization of nascent vessels. Negative regulators (e.g. TSP-1, endostatin) are able to inhibit pro-angiogenic pathways by inhibiting factors such as VEGF. Endostatin, a fragment of collagen XVIII, may also prevent microvascular growth by down regulating proangiogenic and up regulating antiangiogic factors (Kim, Hwang et al. 2002, Abdollahi, Hahnfeldt et al. 2004, Cao 2008). The complex balance of pro- and anti-angiogenic proteins is thought to trigger the growth or regression of capillaries.

\section{VASCULAR ENDOTHELIAL GROWTH FACTOR}

VEGF-A, VEGF-B, VEGF-C and VEGF-D comprise the VEGF family and are recognized by the receptors VEGFR1 (Flt-1), VEGFR2 (KDR/Flk-1) and VEGFR3 (Flt-4) (Robinson and Stringer 2001). VEGF-A, a $\sim 45 \mathrm{kDa}$ protein, is the most biologically important in angiogenesis and is generally referred to as VEGF (Ferrara and Henzel 1989, Hoeben, Landuyt et al. 2004). In the human genome, VEGF has eight exons which are differentially spliced (between 18 and 24_kDa) to VEGF $121, V \mathrm{VGF}_{165}, \mathrm{VEGF}_{189}$, and $\mathrm{VEGF}_{206}$ with the most bioactive form being VEGF $_{165}$ (Tischer, Mitchell et al. 1991, Park, Keller et al. 1993, Roskoski Jr 2007). VEGF mRNA half-life has been reported to be about an hour with an increase reported in response to hypoxia in cell culture (Levy, Levy et al. 1996) and recombinant VEGF has a half-life of $33.7 \pm 13$ minutes in human plasma (Eppler, Combs et al. 2002). With short half-life's, proteins such as heparin sulphate proteoglycans and neuropilin-1 have the ability to prolong VEGF biological activity or enhance receptor binding (Robinson and Stringer 2001). VEGF can also be found bound to the ECM (Ferrara and Henzel 1989, Tischer, Mitchell et al. 1991, Park, Keller et al. 1993). When bound to the ECM, VEGF can be stored until MMP's release the protein allowing it to be biologically active. 
VEGF has long been associated with an increase of vascular permeability of blood vessels, and in fact was originally known as vascular permeability factor (Senger, Connolly et al. 1990, Dvorak 2002). However, the vitality of the gene was demonstrated when attempting to create a total body VEGF KO mouse -where the loss of a single allele was embryonically lethal due to poorly formed vasculature development in utero (Ferrara, Carver-Moore et al. 1996). A myocyte-specific VEGF KO mouse has been successfully generated and displayed a significant reduction in C:F ratio (39\% decrease) and exercise performance, e.g. reduced maximal running speed (by $34 \%$ ) and reduced endurance capacity (by $81 \%$ ) compared to controls (Olfert, Howlett et al. 2009). Furthermore, skeletal muscle capillarity in myocyte-specific VEGF KO mice did not adapt to exercise training as typically seen in exercise trained wild-type mice (Olfert, Howlett et al. 2010). VEGF receptor antagonism also partially inhibits training induced skeletal muscle angiogenesis in ischemia induced hindlimb muscles of mice (Lloyd, Prior et al. 2005). Another study which sequestered VEGF using VEGF Trap prevented increases in skeletal muscle angiogenesis induced by shear stress (e.g. increase blood flow) or muscle stretch/overload conditions (Williams, Cartland et al. 2006). These studies showed that not only is VEGF critical to vascular development in utero but also in response to exercise training.

Short term training ( 7 days) in humans has been positively correlated with an increase of VEGF mRNA in skeletal muscle (Gustafsson, Knutsson et al. 2002). Similar results were reported in rats: VEGF mRNA was elevated after each day of exercise when exercising on a treadmill for one hour a day, for five days (Gavin and Wagner 2001). In another study the effects of detraining were also evaluated in cardiac muscle of rats. Rat VEGF mRNA was investigated in control, trained (10 weeks of treadmill training, 30 hours of total training), and detrained rats (4 weeks). (Marini, Falcieri et al. 2008) VEGF mRNA and cardiac capillary density were elevated after training but those responses returned to control levels after four weeks of detraining (Marini, Falcieri et al. 2008). In a time course study where rats treadmill trained, 
VEGF mRNA had the largest fold change in the white GA, but the SOL and red portion of GA had several time points (3-21 days) at which VEGF mRNA was significantly elevated too (Lloyd, Prior et al. 2003). Although there is a strong trend for an increase in VEGF mRNA and protein in response to exercise, not all studies show this. One example is where participants trained on a bicycle ergometer for four weeks (Hoier, Nordsborg et al. 2012). After cycling, there was an increase of C:F ratio but VEGF protein at basal conditions were not different before or after the training period, but perhaps the window of peak expression/training adaptation had passed by four weeks (Hoier, Nordsborg et al. 2012).

An acute bout of exercise is known to elicit an increase of VEGF mRNA (Olfert, Breen et al. 2001, Hoier, Nordsborg et al. 2012). However, there are mixed results as to whether the acute response remains in a trained and/or detrained state. In a human population that trained on a cycle ergometer for four weeks, VEGF protein was elevated in response to acute exercise both before and after four weeks of training (Hoier, Nordsborg et al. 2012). However, the acute response to exercise in rats that trained for 10 weeks was lost, but returned after 7 days of detraining in the plantaris but not soleus muscle (Malek, Olfert et al. 2010). However, discrepancies between mRNA expression in response to acute exercise may be due to species and fiber type.

\section{MATRIX METALOPROTEINASE -2 AND -9}

There are at least 20 MMP's known to participate in processes such as proteolytic degradation of the ECM and basement membranes (Nagase and Woessner 1999). Degradation is necessary to destabilize current vasculature and ECM allowing for outcroppings of new vessels. However, the activities of MMP's are highly regulated to preserve the integrity of matrix surrounding the vasculature which would be compromised if left unchecked. MMP's are regulated at three key steps. First, DNA must be transcribed into mRNA and translated into an inactive proMMP. Second, proMMP's require enzymatic cleavage to become a mature MMP. 
Third, mature MMP's can be inhibited by tissue inhibitor of metalloproteinase (TIMPS). There are several subclasses of MMP's but one subclass of particular interest is collagenase which harbors the gelatinases: MMP-2 (collagenase A or pro-MMP, 72kDa; active MMP-2, 62kDa) and MMP-9 (collagenase B, 92kDa; active MMP-9 82kDa) (Schmalfeldt, Prechtel et al. 2001, Shimokawa, Katayama et al. 2002). Gelatinases are instrumental in angiogenesis due to their ability to degrade the most prevalent protein in skeletal muscle basal lamina: collagen IV.

There is strong evidence that MMP-2 and MMP-9 are critical for angioadaptation. MMP2 has been localized to the ECM around muscle fibers, capillaries, and inside the muscle fiber (Rullman, Norrbom et al. 2009) and is involved with cell fusion (Zimowska, Brzoska et al. 2008). Pharmacological inhibition of MMP activity prevents capillary adaptation in chronic electrically stimulated hindlimb muscles, demonstrating that MMP activity is required for skeletal muscle to undergo capillarity adaptation in response to chronic muscle contractions (Haas, Milkiewicz et al. 2000). MMP-9 is localized to capillaries and around muscle fibers without expression in muscle fibers (Rullman, Norrbom et al. 2009), may be involved in myoblast cell differentiation (Zimowska, Brzoska et al. 2008), plays a role in collagen assembly and compaction (Whatling, McPheat et al. 2004) and inhibition of MMP-9 inhibits endothelial cell migration (Jadhav, Chigurupati et al. 2004). Both MMP-2 and MMP-9 are more abundant in zones where cellular differentiation occurs and participate in smooth muscle cell migration and neointima growth (Whatling, McPheat et al. 2004). The activity of MMP's may be, in part, dependent on the angiogenic stimulus. For example MMP-2 protein levels can be increased with muscle stretch by unilateral extirpation of the tibalis anterior but not in response to an increase in circulating blood flow and increases in shear stress induced by prazosin (Rivilis, Milkiewicz et al. 2002). The same authors reported no significant change of MMP-9 under either condition

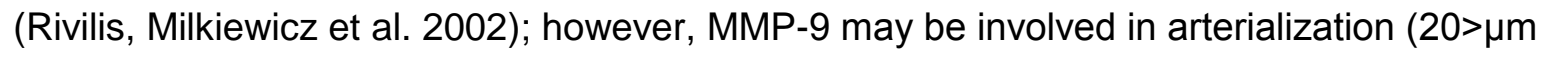
diameter) with administration of prazosin (van Gieson and Skalak 2001). 
The effects of a protein can be observed when knocking it out in a mouse model. MMP-2 and MMP-9 null mice both are fertile but subtle differences were noted. MMP-2 KO mice tended to be about $15 \%$ smaller than littermates (Itoh, Ikeda et al. 1997) and had a slower angiogenic response and tumor growth rate than littermate controls (Itoh, Tanioka et al. 1998). MMP-9 KO mice showed delayed angiogenesis in bone growth plates (Itoh, Ikeda et al. 1997), did not stop tumor growth (Masson, de la Ballina et al. 2005), and had slower smooth muscle cell proliferation and migration in arteries (Cho and Reidy 2002). Interestingly, knocking out MMP-2 or MMP-9 individually was not sufficient to arrest tumor growth/angiogenesis but when both were knocked out in the same mouse, tumor growth was halted (Masson, de la Ballina et al. 2005), possibly implicating that they work together. However, other studies make convincing arguments that MMP-9 appears to be a larger contributor and possibly a switch that triggers the activation of quiescent vasculature (Bergers, Brekken et al. 2000, Bergers and Benjamin 2003); whereas MMP-2 may play a minor role in growth of new vasculature (Bergers, Brekken et al. 2000).

MMP-2 and MMP-9 have different expression profiles and have been evaluated under basal conditions and in response to acute exercise but the temporal response is still unclear. Under basal conditions in mouse skeletal muscle, MMP-2, but not MMP-9, mRNA and protein is normally expressed (Kherif, Lafuma et al. 1999). With acute exercise, one study saw a decrease of MMP-2 proMMP-2 immediately after exercise (Koskinen, Heinemeier et al. 2004), another study reported an increase of MMP-2 protein only at 30 min post cycling (Suhr, Brixius et al. 2007), a third study saw no increase of protein under basal conditions in long and short track athletes pre season but reported an increase 1 hour post acute exercise in long track athletes (Suhr, Rosenwick et al. 2010). MMP-2 was evaluated up to 3 days after acute exercise on a treadmill, levels were low immediately and 1 day after exercise but were elevated 3 days post exercise (Koskinen, Heinemeier et al. 2004). Meanwhile, MMP-9 has been reported to be elevated 0-4 hours post acute exercise (Koskinen, Heinemeier et al. 2004, Suhr, Brixius et al. 
2007, Suhr, Rosenwick et al. 2010) and remained elevated at 1 and 3 days post acute exercise (Koskinen, Heinemeier et al. 2004). In response to a season of training, MMP-9 serum levels tended to be lower in both short and long track athletes under basal conditions and after acute exercise, but MMP-2 levels were not affected by training (6 months) (Suhr, Rosenwick et al. 2010). In short, MMP-2's response to acute exercise has yet to be fully understood but MMP-9 has an immediate increase of protein levels that remain for at least 3 days but dissipates with chronic training.

There have been a number of articles published in a human model about MMP-2 and MMP-9 protein expression in response to acute exercise but there are fewer articles about chronic exercise. One study reported ten days of high intensity running elevated MMP-9 mRNA in rat GA and SOL (Carmeli, Haimovitz et al. 2007). Single leg training in humans, also resulted in an increase of MMP-9 mRNA taken from a muscle biopsy at 10 and 35 days, but MMP-9 protein remained unchanged (Rullman, Norrbom et al. 2009). In the same single leg study, MMP-2 mRNA levels were significantly elevated after 10 and 35 days of training but only had increased protein levels at day 10 (Rullman, Norrbom et al. 2009). These two studies suggest that MMP-2 mRNA is elevated with increasing exercise duration where as MMP-9 is not. Many of the studies evaluated serum and plasma levels but examination of protein expression in the skeletal muscle where angiogenesis occurs is needed.

\section{NUCLEOLIN}

Nucleolin (formally known as C23) is a 105 kDa nuclear multifunctional protein which plays a role in many different cellular actions including cell proliferation, ribosome biogenesis, mitogenesis, adhesion, and differentiation (Andersen, Lam et al. 2005, Destouches, El Khoury et al. 2008). Furthermore, nucleolin, a major nuclear protein in cells that proliferate exponentially (Lapeyre, Bourbon et al. 1987), also serves as a shuttle between the nucleus, cytoplasm, and cell surface (Borer, Lehner et al. 1989, Schmidt-Zachmann, Dargemont et al. 1993, 
Hovanessian, Soundaramourty et al. 2010), binds to DNA with high affinity (Chen, Chiang et al. 1991), can control the unwinding of DNA and RNA duplexes (Tuteja, Huang et al. 1995), and angiogenesis (Destouches, El Khoury et al. 2008). Nucleolin is exclusively a self cleaving fragment (cleaved at 6 hours, completely degraded at 24 hours) but is inhibited by an unidentified proteolytic inhibitor in the nuclei (Chen, Chiang et al. 1991). The unidentified inhibitor is negatively charged and binds to an enzymatic domain found in the two thirds side of the C-terminal (Fang and Yeh 1993). When using agonist HB-19 pseudopeptide which binds to the C-terminal of cell surface nucleolin, tumor growth and angiogenesis has been halted both in vivo and in vitro with no toxicity to normal tissue (Destouches, El Khoury et al. 2008). Inhibition of nucleolin in cell culture inhibited migration but did not affect proliferation or adhesion (Huang, Shi et al. 2006). The presence of nucleolin on the cell surface is highly correlated with cell growth conditions in cell culture (Hovanessian, Soundaramourty et al. 2010); degraded nucleolin quantities gradually decrease as cells start to become more proliferative (Chen, Chiang et al. 1991). Nucleolin, with its strong correlation to cell proliferation, has been used as a marker of angiogenic endothelial cells in tumor blood vessels (Christian, Pilch et al. 2003). When antinucleolin antibody was injected into mice, the antibody only accumulated on the surface of tumor blood vessels (Shi, Huang et al. 2007). These points emphasize the close connection between nucleolin expression and angiogenesis, indicating nucleolin might serve as a critical angiogenic biomarker for proliferating endothelial cells.

Due to the strong correlation of nucleolin and angiogenesis and a lack of articles evaluating it in response to acute exercise or training, we believe that further evaluation of the protein in skeletal muscle angiogenesis is warranted.

\section{ENDOSTATIN}

Endostatin is a $22 \mathrm{kDa}$ anti-angiogenic C-terminal fragment of collagen XVIII found throughout the body with most of the monomer being prevalent in the vascular and epithelial 
basal membrane around muscular structures (Faye, Chautard et al. 2009). Endostatin is an effective inhibitor of angiogenesis by restricting endothelial cell proliferation and migration by inducing apoptosis, down-regulating pro-angiogenic signaling pathways and up-regulating antiangiogenic genes (Kim, Hwang et al. 2002, Abdollahi, Hahnfeldt et al. 2004, Cao 2008). Since endostatin is derived from collagen matrix, its formation is dependent not on transcription but by other regulators such as MMP-9, elastase, and cathepsin $L$ which cleave the fragment from collagen XVIII (Wen, Moses et al. 1999, Felbor, Dreier et al. 2000, Chang, Javier et al. 2005, Heljasvaara, Nyberg et al. 2005). MMP's known to produce the endostatin fragment are MMP-3, $-7,-9,-13,-20$, whereas MMP-1, -2, -8 and -12 do not (Heljasvaara, Nyberg et al. 2005). A cell culture study suggests that generation of endostatin may be a two step process. The first step is metal dependent, which trims collagen XVIII to NC1 [69]. The second step of endostatin generation is elastase dependent, elastase cleaves NC1 to endostatin (Wen, Moses et al. 1999). Endostatin KO mice, also known as Col18a1 KO mice, do not have an abnormal increase of capillarity in most organs and tumor growth is not increased in these mice, however aortic implants from KO mice into WT mice had increased microvessel growth (Li and Olsen 2004) the mice may also have ocular abnormalities due to abnormal retinal vessel growth (Marneros and Olsen 2005).

The endostatin fragment works by blocking general mechanisms that influence endothelial cell growth including interfering with VEGFR2 receptors (Kim, Hwang et al. 2002, Lee, Jang et al. 2002, Cao 2008). An exception to its antiangiogenic activity would be during embryonic development where it may operate in an angio-modulatory way. (Kim, Hwang et al. 2002, Schmidt, Wenzel et al. 2004, Cao 2008) Interestingly, endostatin does not act solely by inhibiting pro-angiogenic proteins but also up regulates anti-angiogenic proteins (Abdollahi, Hahnfeldt et al. 2004). For example, endostatin can up regulate TSP-1 and simultaneously down regulating the TSP-1 suppressor ID-1 (Volpert, Pili et al. 2002). Endostatin does more than just modulate TSP-1, it is capable of affecting a wide range of proteins. DNA and antibody 
array technology have shown that endostatin, when administered as a drug, is capable of inhibiting 65 varieties of tumor and modified $12 \%$ of the human genome in human dermal microvascular endothelial cells (mostly angiogenic pathways) (Abdollahi, Hahnfeldt et al. 2004). Endostatin concentrations appear to vary due to tissue oxidative capacity. Under basal conditions, the heart has 4.9 times lower endostatin and 1.5 higher VEGF protein level than the anterior tibialis, which correlates with the heart having a greater capillarity density. However after rats exercised for three weeks, there was nearly a 3 fold decrease of endostatin and 2 fold increase of VEGF in the TA (Gu, Shparago et al. 2006).

Endostatin has been well characterized in tumor research but there are limited articles about its regulation and expression in skeletal muscle in response to exercise. During acute exercise, circulating levels of endostatin increased in a linear relationship to peak $\mathrm{VO}_{2}$ $\left(\mathrm{R}^{2}=.9388\right)(\mathrm{Gu}$, Gadonski et al. 2004), but exercise did not increase endostatin levels in plasma with increasing time exercising at $50-65 \%$ of $\mathrm{VO}_{2} \max$ (Rullman, Rundqvist et al. 2007). In response to acute exercise, endostatin is significantly elevated at 0 hours post exercise(Suhr, Brixius et al. 2007). Endostatin levels were reduced after training basally and in response to acute exercise in both elite short and long track athletes (Suhr, Rosenwick et al. 2010). Another study measured VEGF and endostatin concentrations pre and post 6 months of training and found VEGF levels were not affected but training significantly reduced endostatin levels (Brixius, Schoenberger et al. 2008). In short, endostatin plasma levels seem to be elevated in response to acute exercise: the harder the workload (working at a higher VO2), the higher the endostatin level. These studies imply that endostatin correlates with intensity $\left(\mathrm{VO}_{2}\right)$ of acute exercise and decreases with chronic training, but the temporal response of endostatin to training and detraining are still poorly characterized. 


\section{THROMOBSPONDIN-1}

TSP-1 is a large, $450 \mathrm{kDa}$ trimeric, extracellular matrix glycoprotein. It is produced by several different cell types including $\alpha$-granules, smooth muscle cells, endothelial cells, fibroblasts, kerationocytes, neutrophils and macrophages (Duffield 2003, Olfert and Birot 2011). TSP-1 is effective by inhibiting endothelial cell proliferation, adhesion, and motility (Bagavandoss and Wilks 1990, Taraboletti, Roberts et al. 1990). TSP-1 can inhibit the angiogenic response by binding to CD47 which in turn inhibits VEGFR2's phosphorylation and thus downstream targets (Isenberg, Frazier et al. 2008, Kaur, Martin-Manso et al. 2010). The protein may not be needed in large quantities to inhibit angiogenesis under basal conditions, as pM concentrations in plasma appears to be sufficient (Isenberg, Frazier et al. 2008).

The first mouse strain used to produce TSP-1 KO mice were 129Sv background mice, which exhibited increased levels of white blood cells, lordotic curvature of the spine, and had lung abnormalities making them susceptible to pneumonia (Lawler, Sunday et al. 1998). This phenotype has not manifested in C57Blk6 strain of TSP-1 KO mice which are currently commercially available. Recent evidence have found the C57Blk6 TSP-1 KO to have greater microvessel density compared to wild-type mice under basal conditions (Fitchev, Wcislak et al. 2010). These TSP-1 KO mice also had $67 \%$ increased in endurance running test and an $11 \%$ increase in maximal running speed, which can likely be attributed to significant increase of skeletal and cardiac muscle capillarity (Malek and Olfert 2009).

There are only a few studies that examine TSP-1 in response to exercise or training. TSP-1 mRNA expression was examined after training rats for one hour a day up to five days. The study found that mRNA was elevated from basal conditions only on days one and two, and returned to baseline conditions on days 3-5 (Olfert, Breen et al. 2006). After chronic exercise consisting of four weeks of bicycle ergometer training, an increase of C:F ratio was reported but VEGF and TSP-1 protein at basal conditions were not remarkable before and after the training period (Hoier, Nordsborg et al. 2012). The lack of TSP-1 response may be due to the time 
points selected for samples to be taken, for example, peak expression may have been at day 3 which fell between the 0 and 4 week time points. The cycling study also examined acute response to exercise under basal and trained states and showed TSP-1 mRNA had an acute response at one and three hours post exercise under basal and trained conditions (Hoier, Nordsborg et al. 2012). Finally, TSP-1 expression can be altered with other compounds such as (-)-epicatchin found in cacao. When (-)-epicatchin was administered in mouse drinking water, TSP-1's response following training was blunted preventing a loss of capillarity; TSP-1 levels remained at a trained state even though mice were detraining (Hüttemann, Lee et al. 2011). Angiogenesis research has largely examined proangiogenic proteins but antiangiogenic proteins including TSP-1 may also play an equally important of a role in regulating capillary growth and development and therefore their response need to better characterized.

\section{INTEGRATED ANGIOGENIC PROTEIN RESPONSE TO EXERCISE}

Collectively the review of VEGF, MMP-2, MMP-9, nucleolin, endostatin, and TSP-1 highlight their individual importance in the angiogenic process, however, there is a deficiency of knowledge of how these proteins respond to physiologic stimuli that alter vascular networks such as acute exercise, training and/or detraining. The current proposal examines the temporal expression (basal and time course response to acute exercise) of these factors in conjunction with morphological changes in skeletal muscle capillarity in mice at differing time points up to 28 days of voluntary exercise training, and at various time points up to 28 days after detraining, in order to gain further insight in the temporal regulation of capillary expansion and rarefaction. But it also is important to note that these factors may interact and influence each other. Below is as summary of the known effects of each factor with one another, or other potential angioregulatory factors. Identifying the potential temporal associations or links between these individual angiogenic may help to more clearly understand the physiologic regulation of exercise-induced skeletal muscle angiogenesis. 
VEGF:

- VEGF is capable of activating MMP-2 in isolated endothelial cells (Rivilis, Milkiewicz et al. 2002), and inhibition of MMP-2 by RNAi decreased VEGF protein (by decreasing integrin $\alpha \mathrm{V} \beta 3$ mediated inhibition of the PI3k/AKT pathway)(Chetty, Lakka et al. 2010).

- $\quad$ MMP-9 is capable of releasing angiogenic factors including VEGF making them more bioavailable (Bergers, Brekken et al. 2000, Giraudo, Inoue et al. 2004, Lee, Jilani et al. 2005) and in a positive feedback mechanism, VEGF can also up regulate MMP-9 (Wang and Keiser 1998, Giraudo, Inoue et al. 2004).

- VEGF mediates nucleolin mobilization from the nucleus to the endothelial cell surface; without VEGF, nucleolin quickly disappears from the cell surface (Huang, Shi et al. 2006).

- Endostatin levels in TA have been reported to be inversely correlated with capillary density and VEGF levels (Gu, Shparago et al. 2006). Furthermore, several different models have shown that endostatin in capable of inhibiting VEGF (Yamaguchi, AnandApte et al. 1999, Wang, Xu et al. 2007, Hu, Xia et al. 2012). One method includes inhibiting the KDR/Flk1 VEGF receptor (Kim, Hwang et al. 2002) and another may be mediation of endostatin and VEGF by nucleolin. (Huang, Shi et al. 2006, Shi, Huang et al. 2007)

- $\quad \underline{T S P-1}$ can interact directly to inhibit VEGF by ligating the VEGFR2 receptor and inhibiting phosphorlation, It can also displace VEGF from a mutual heparin binding site on endothelial cells (Gupta, Gupta et al. 1999)

Nucleolin:

- The interaction between nucleolin and endostatin could be a crucial element for cell proliferation and ultimately angiogenesis; heparin binding site of endostatin is critical for 
nucleolin to bind (Shi, Huang et al. 2007). Nucleolin and endostatin are often found colocalized on the cell surface but once endostatin is transported to the nuclei via nucleolin, endostatin phosphorylates nucleolin inhibiting its role in cell proliferation (Shi, Huang et al. 2007).

- The pseudopeptide HB-19 which inhibits cell surface nucleolin also causes a down regulation of VEGF and $\underline{\text { MMP-2 }}$ in cell culture and ultimately suppress tumor growth (Krust, El Khoury et al. 2011).

- Nucleolin is capable of binding to the 3' region of MMP-9 mRNA, stabilizing it for translation and leading to an increase in the degradation of ECM but does not appear to participate in the same binding function for MMP-2 (Fähling, Steege et al. 2005).

MMP-2 and MMP-9

- Both are gelatinases but have their own identity. When knocking out MMP-2 or MMP-9 individually, neither was individually able to stop tumor growth/angiogenesis but when both of these MMP's were knocked out in the same mouse, tumor growth was halted (Masson, de la Ballina et al. 2005)

- Endostatin may be cleaved by several MMP's including MMP-9 (Heljasvaara, Nyberg et al. 2005). Endostatin inhibits activity of proMMP-2 (Kim, Hwang et al. 2002).

- TSP-1 has been shown to increase MMP-2 (Lee, Esemuede et al. 2003) and MMP-9 (Albo, Shinohara et al. 2002) activation in cell culture.

\section{Endostatin:}

- $\quad \underline{T S P-1}$ shares common cell surface binding ligands with endostatin: $\alpha 5 \beta 1$ and $\alpha v \beta 3$ (Sipes, Krutzsch et al. 1999, Rehn, Veikkola et al. 2001). Furthermore, endostatin can up regulate TSP-1 and simultaneously down regulating the TSP-1 suppressor ID-1 (Volpert, Pili et al. 2002). 
It can be concluded that VEGF, MMP-2, MMP-9, nucleolin, endostatin and TSP-1 all play a role in angiogenesis and often have a relationship with each other. Much of the inference between proteins has been implicated by cell culture and tumor research, it has yet to be elucidated if similar associations occur in response to exercise-induced skeletal adaptation. The current proposal seeks to better understand the relationship between these factors by examining their temporal response to acute exercise, chronic exercise and physical deconditioning. 
AIMS:

Specific Aim 1: Examine the temporal expression of key positive and negative angiogenic regulators in skeletal muscle in response to acute exercise.

H1.1: We hypothesize that both positive (e.g. VEGF, MMP-2, MMP-9, nucleolin) and negative (e.g. TSP-1, endostatin) angiogenic factors will be elevated in response to a single bout of exercise.

Specific Aim 2: Examine the temporal expression of key positive and negative angiogenic regulators in skeletal muscle in response to training as well as the responsiveness of the factors to acute exercise immediately following training.

$\mathrm{H}$ 2.1: We hypothesize the basal expression of positive factors will remain elevated, while basal expression of negative factors will decrease, with increasing temporal exposure to training. $\mathrm{H}$ 2.2: We hypothesize the acute exercise response of positive, but not negative, angiogenic regulators will be blunted or lost following exercise training.

Specific Aim 3: Examine the temporal expression of key positive and negative angiogenic regulators in skeletal muscle in response to detraining and as well as the responsiveness of these factors to acute exercise following detraining.

H3.1: We hypothesize there will be an increase in negative, relative to positive, angiogenic factors with detraining.

H3.2: We hypothesize the blunted acute response of positive angiogenic regulators to exercise following training will return after detraining.

H3.3: We hypothesize that oxidative muscles will have greater changes in angiogenic regulators (both positive and negative) compared to glycolytic muscles. 
CHAPTER 2:

Temporal response of positive and negative regulators in response to acute and chronic exercise training

Sara A. Olenich ${ }^{1}$, Navarre Gutierrez-Reed ${ }^{2}$, and I. Mark Olfert ${ }^{1}$

(1)West Virginia University School of Medicine

Center for Cardiovascular and Respiratory Sciences

Division of Exercise Physiology

One Medical Center Dr.

Morgantown, WV 26506-9105

(2)University of California, San Diego

Department of Medicine

Division of Physiology

9500 Gilman Drive

La Jolla, CA 92093-0623

Short Title: Angiogenic temporal responses to training

Total words: 6151

Corresponding Author:

Mark Olfert, Ph.D.

West Virginia University School of Medicine

Center for Cardiovascular and Respiratory Sciences

Division of Exercise Physiology

One Medical Center Dr.

Morgantown, WV 26506-9105

Tel 304-293-7597

Fax 304-293-5513

molfert@hsc.wvu.edu

THIS PAPER IS CURRENTLY UNDER REVIEW AT THE JOURNAL OFPHYSIOLOGY. SUBMITTED ON MARCH 13, 2013. 


\section{Abstract (292)}

Angiogenesis is controlled by a balance between positive and negative angiogenic factors, but temporal protein expression of many key angiogenic regulators in response to exercise are still poorly defined. We evaluated the skeletal muscle temporal expression of proangiogenic vascular endothelial growth factor (VEGF), matrix metalloproteinases (MMP)-2, MMP-9, and nucleolin; as well as the anti-angiogenic factors thrombspondin-1 (TSP-1) and endostatin, in response to acute and chronic exercise involving 3, 5, 7, 14, or 28 (d)ays of voluntary wheel run training. Protein expression of VEGF, endostatin and nucleolin were increased 2-4 hours after a single exercise bout; whereas MMP-2 was elevated between a 1224 hour window $(p<0.05)$. In response to training, muscle capillarity was increased after $7 \mathrm{~d}, 14 \mathrm{~d}$ and 28d training $(p<0.01)$. Surprisingly, basal VEGF and MMP-2 were significantly lower with $3 d$ training, and basal MMP-9 was lower with $5 d, 7 d$ and $14 d$ training $(p<0.05)$. However, $7 d$ training significantly increased basal VEGF and MMP-2 compared to $3 d$ training $(p<0.05)$. Basal muscle TSP-1 in 14d and 28d trained mice were significantly greater compared to $5 d$ and $7 d$ trained $(p<0.05)$, and tended to increase compared to basal control levels $(p<0.10)$. The acute responsiveness of VEGF (as seen in untrained mice) was lost after $7 d$ training. Taken together, these data support the notion that skeletal muscle angiogenesis is controlled by a balance between positive and negative mitogens, and reveals a complex, highly-coordinated, temporal scheme whereby these factors can differentially influence capillary growth in response to acute versus chronic exercise. Given the multitude of angioregulatory factors that may be involved in regulating skeletal muscle angiogenesis, additional studies examining the temporal response of each potential angiogenic factor will be necessary before a full understanding of the mechanisms regulating exercise-induced skeletal muscle angiogenesis will be gained. 


\section{Introduction}

High metabolic demands imposed by exercise on skeletal muscle during physical activity are known to elicit cardiovascular adaptations. Among the adaptations induced by exercise training is the expansion of skeletal muscle vascularity, which is recognized to be an important means of increasing conductance of oxygen within the exercising muscle (Snyder, 1987; Snyder

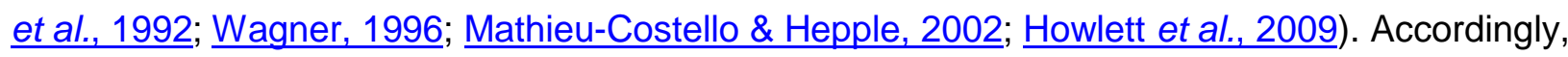
there has been much interest in identifying the factors and mechanisms regulating skeletal muscle angiogenesis in response to exercise (see reviews by (uudlicka et al., 1992; Prior et al.. 2004; Bloor, 2005; Egginton, 2009)).

Angiogenesis is a complex process thought to be regulated by a balance of proangiogenic and anti-angiogenic factors. Among the pro-angiogenic factors known to regulate skeletal muscle angiogenesis, are vascular endothelial growth factor (VEGF) and matrix metalloproteinases -2 and -9 (MMP-2, MMP-9), which have all been shown to be essential in the angiogenic response to physical activity. For example, VEGF receptor antagonism partially inhibits training induced skeletal muscle angiogenesis in ischemic hindlimb muscles of mice (Lloyd et al., 2005) and sequestering circulating VEGF (using VEGF Trap) prevents increases in skeletal muscle angiogenesis induced by elevated shear stress or muscle stretch/overload conditions (Williams et al., 2006). We have recently shown that skeletal muscle angioadaptation to 6-weeks of high intensity treadmill training is absent in healthy myocyte VEGF-deficient mice (Olfert et al., 2010), demonstrating that myocyte-derived VEGF is required for exercise-induced skeletal muscle angiogenesis. Likewise, there is evidence that MMP's are equally important and necessary for exercise-induced angioadaptation. Haas et al. (2000) have shown that pharmacological inhibition of MMP activity prevents capillary adaptation in chronic electrically stimulated hindlimb muscles, establishing that MMP activity is also required for skeletal muscle to undergo capillarity adaptation in response to chronic muscle contractions. Collectively these 
studies highlight the importance of VEGF and MMP activity in the regulation of training-induced skeletal muscle capillary adaptation.

Recently, anti-angiogenic factors have also been shown to be stimulated by exercise. For example endostatin and thrombospondin-1 (TSP-1) are increased in response to acute exercise (Gu et al., 2004; Olfert et al., 2006). Mice lacking TSP-1 also display a greater basal muscle capillarity and increase exercise performance compared to wild-type mice (Malek \& Olfert, 2009). Circulating endostatin has been shown to increase with acute exercise (Gu et al.. 2004), but is reduced after prolonged training in humans (Brixius et al., 2008; Suhr et al., 2010). Recent evidence suggests that the anti-angiogenic action of endostatin on endothelial cell activity may be dependent on the nuclear protein nucleolin (Shi et al., 2007). Nucleolin has been previously used as a marker of endothelial cell proliferation for cells undergoing

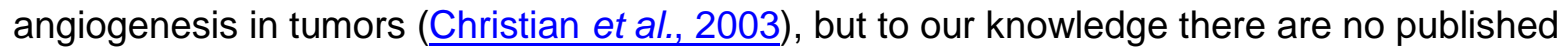
data on the effects of exercise on nucleolin expression. Given that nucleolin is involved in transcription processing, editing and assembly of ribosomes and import/export of proteins from the cytoplasm to the nucleus (Srivastava \& Pollard, 1999), it is conceivable that it may be altered or be responsive to exercise. Supporting this this idea, is circumstantial evidence that nucleolin may interact with angiogenic mitogens, such as VEGF (ㅂuang et al., 2006), bind to the 3' region of MMP-9 stabilizing it for translation (Fahling et al., 2005), and interact with endostatin when translocated to the cell surface (Shi et al., 2007). Thus, we have also sought to examine whether or not acute and/or chronic exercise might alter nucleolin expression.

Several studies have reported the temporal transcriptional/gene expression responses of VEGF and MMPs (as well as other angiogenic mitogens) to acute and chronic exercise (Breen et al., 1996; Brown \& Hudlicka, 2003; Lloyd et al., 2003; Gustafsson et al., 2005), but few studies have done so at the translational (i.e. protein) level. Understanding protein level changes in response to acute and chronic exercise is vitally important since it is known that 
transcriptionally-mediated events do not always translate to protein biosynthesis. Moreover, factors like endostatin are not gene encoded peptides, but rather formed as a cleavage fragment from other molecules (e.g. endostatin is a 22-kd C-terminal fragment formed from collagen XVIII). Therefore, any response from endostatin to exercise, or in response to any stimulus, would not be detectable at the transcriptional level. Additionally, given the growing number of factors thought to regulate angiogenesis it is unlikely that the temporal protein responses to an angiogenic stimulus (such as exercise) would occur at one time point. Given the coordination of factors and events needed for successful control of angiogenesis, we sought to examine the temporal protein expression of several key angiogenic regulators (VEGF, MMP2, MMP-9, and nucleolin) in response to acute exercise, as well as at varying time points following chronic exercise training. We include the anti-angiogenic regulators (TSP-1 and endostatin) in conjunction with morphological assessment of skeletal muscle capillarity, to better understand the dynamic balance between several key positive and negative angiogenic regulators that are believed to significantly influence skeletal muscle angiogenesis. Specifically, we are testing the hypothesis that negative angiogenic regulators (such as endostatin and TSP1) are differentially expressed in response to acute versus chronic exercise, such that the actions of positive angiogenic molecules (VEGF, MMPs, and possibly nucleolin) are inhibited with acute, but not chronic, exercise.

\section{Materials and Methods}

Mice: All mice were purchased from Jackson Laboratories (Bar Harbor, ME) and housed in a pathogen-free vivarium room with $12 \mathrm{hr}: 12 \mathrm{hr}$ light:dark cycle. Mice were fed rodent chow (Harlan Tekland 8604, Madison, WI) and tap water ad libitum. Animal care and handling were performed in accordance to PHS Animal Welfare Act and received approval by the West 
Virginia University and University of California-San Diego Institutional Animal Care and Use Committees.

Study 1 - Time course response to single bout of acute exercise: Twenty-four male C57BI/6 mice were randomly assigned to either a control group (no exercise, $n=4$ ) or one of seven acutely exercised groups ( $n=4 /$ group). Following the 1 -hour of acute exercise at 20 $\mathrm{m} / \mathrm{min}, 10^{\circ}$ incline on a rodent treadmill (model CL-4, Omnitech, Columbus, $\mathrm{OH}$ ), hindlimb gastrocnemius muscle was removed at either 0 (immediately post exercise), 2, 4, 6, 12, or 24 hours post exercise from deeply anesthetized mice (details for tissue harvesting are provided below).

Study 2 - Temporal response of angiogenic regulators to exercise training: Seventy-two male C57BI/6 mice were randomly assigned into a control (no training, $n=12$ ) or one of five training groups ( $n=12 /$ group). Training mice had free access to cage running wheels (4.5" Mouse Wheel, Respironics, Bend, OR) for either 3, 5, 7, 14, or 28 days. During wheel training mice were individually housed and wheel activity (i.e. distance, running time, mean running velocity, and maximum running velocity) was monitored and recorded every 24 hours using digital bike computers (Cateye Strada CC-RD100N, CatEye North America, Boulder, CO).

Following training, wheels were removed from the cage, and mice were given a 24-hour exercise washout period prior to assessment of either basal or acute exercise responses. After the 24-hour exercise washout, half of the mice in each group were randomly selected to perform a 1-hour treadmill acute exercise $(n=6)$ while the remaining mice served as basal controls $(n=6)$. This allowed for examination of the responsiveness of angiogenic proteins to exercise following different days of training. For mice acutely exercised, the gastrocnemius muscle was surgically removed 4 hours after the acute exercise bout (1-hour at $20 \mathrm{~m} / \mathrm{min}, 10$ degree incline). The 4hour time point was selected based on the peak responses observed from Study 1. For all mice, 
muscles from the left leg were used for molecular analysis and muscles from the right leg were used for morphometric analysis.

Tissue collection and processing: All mice were anesthetized with sodium pentobarbital (Nembutal, $50 \mathrm{mg} / \mathrm{kg}$ ) via intraperitoneal injection. Once anesthetized the gastrocnemius muscle from the left leg was carefully removed at its respective origin and insertion attachment, weighed, and immediately flash frozen in liquid nitrogen for protein analyses. The gastrocnemius from the right leg was removed in the same manner, positioned transversely on a cork base, carefully embedded in tissue freezing medium (TBS Tissue Freezing Medium ${ }^{\text {TM }}$, Triangle Biomedical Sciences, Durham, NC, USA) and immediately flash-frozen in liquid nitrogen cooled isopentane (2-methylbutane, Fisher Chemical O3551-4, Fisher Scientific). All muscle samples were stored at $-80^{\circ} \mathrm{C}$ until processed for molecular or morphometric analyses.

Protein analysis: Skeletal muscle was homogenized using a lysis buffer containing 50 $\mathrm{mM}$ Tris/ $\mathrm{HCl}(\mathrm{pH} 7.4), 150 \mathrm{mM} \mathrm{NaCl}, 0.5 \%$ Triton X-100, and protease inhibitors (Complete ${ }^{\mathrm{TM}}$ Tablet, Roche Applied Science, Indianapolis, IN). Homogenates were centrifuged at $4^{\circ} \mathrm{C}, 8000 \mathrm{~g}$ for 10 minutes and supernatants removed and placed in new tubes. Total protein was measured by the bicinchoninic acid method (BCA protein assay kit, Bio-Rad Laboratories, Hercules, CA).

Mouse VEGF, MMP-2, and MMP-9 protein levels were measured from $150 \mu \mathrm{g}$ of total protein using a commercially available ELISA kits (VEGF \#MMV00; MMP-2 \#DMP2F0; MMP-9 \#MMPT90; from R\&D Systems, Minneapolis, MN). All analyses were performed according to the manufacturer's specification and optical density was measured using a microplate reader at $450 \mathrm{~nm}$ corrected by readings at $540 \mathrm{~nm}$ (Bio Rad Model 550, Global Medical Instrumentation Inc., Ramsey, Minnesota). TSP-1, endostatin and nucleolin were analyzed using Western

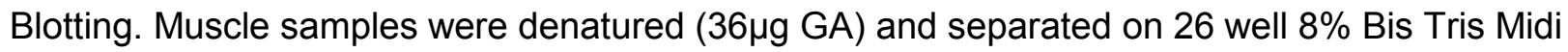
Gels (Novex WG1003BX10, Carlsbad, CA) and blotted onto a $0.45 \mu \mathrm{m}$ nitrocellulose membrane 
(nitrocellulose membrane \#88018, Thermo Scientific, Rockford, IL). The membrane was blocked with 5\% fat-free milk in TBS and probed with respective antibodies TSP-1 (Thrombospondin-1, A6.1, sc-59887, Santa Cruz Biotechnology, Santa Cruz, CA); nucleolin (nucleolin antibody \#A300-711A, Bethyl Laboratories, Montgomery, TX); and endostatin (anti-mouse endostatin antibody \#AF570 R\&D Systems, Minneapolis, MN). The housekeeping genes $\beta$-tubulin (\#4970 Cell Signaling Technology) and GAPDH (mouse anti-GAPDH \#39-8600, Invitrogen, Camarillo, CA) were used to normalize the respective levels of TSP-1, endostatin, and nucleolin measured in order to account for potential variations in total protein loaded within each gel.

Chemiluminescence detection allowed for visualization of proteins (Pierce ECL Western Blotting Substrate, \#32209, Rockford, IL), images of blots were taken using Genesnap software (version 7.01; Syngene). Protein expression was quantified by using NIH ImageJ Software (version 1.62) and expressed as densitometric arbitrary units (A.U.). The same control samples were always loaded on every gel in order to allow normalization of densitometry across blots and allow all groups to be compared relative to each other.

Histochemistry. Frozen muscles embedded in tissue freezing medium were cut into serial $10 \mu \mathrm{m}$ transverse sections using a cyrotome at $-20^{\circ} \mathrm{C}$ (Reichert Jung Cryocut 1800 ; Cambridge Instruments, Buffalo, NY) and mounted on glass slides (Fisher Superfrost \#12-5447, Fisher Scientific) for histochemical analysis of muscle morphometry. Great care was taken to ensure that the widest part of the muscle was sectioned and that cuts were made perpendicular to the orientation of the fibers. Muscle capillarity was determined by measuring capillary-to-fiber ratio $(\mathrm{C}: \mathrm{F})$ from alkaline phosphatase and dipeptidylpeptidase IV stained sections (Mrazkova et al., 1986). Stained muscle sections were viewed by light microscopy and digitally imaged at $25 x$ magnification by a researcher blinded to the experimental group identity. Muscle capillarity was assessed from deep and superficial regions in both lateral and medial gastrocnemius. Images were obtained in a non-overlapping checkerboard manner within each of the 4 quadrants (i.e. 
lateral head superficial, medial head superficial, lateral head deep, medial head deep). This resulted in an average of $771 \pm 123(\mathrm{SD})$ muscle fibers being analyzed from each animal.

Statistical Analyses: All data shown are mean \pm SEM, unless otherwise indicated. Changes in protein expression, exercise performance during training (i.e. daily distance run, time running, average speed and maximal speed), and muscle capillarity (i.e. C:F) were analyzed using ANOVA. The combined effects of training (i.e. groups trained for different days) and the angiogenic factor response to acute exercise (i.e. basal vs. acute exercise) for proteins were separately determined using a $2 \times 2$ factorial ANOVA. When a main effect was observed, post hoc testing with a Fisher's PLSD was used to examine group differences. All statistics were performed using Statview Statistical Software package (v5.0.01, SAS Institute Inc., Cary, NC). Significance was set at $\alpha$ level of $p=0.05$.

\section{Results}

Study 1 - Time course response to single bout of acute exercise. Age, body and muscle masses of mice were not different in any of the respective groups for these mice (Table 1). Expression of skeletal muscle VEGF, endostatin and nucleolin protein levels were significantly greater than control at 2 and 4 hours post exercise $(p<0.05$, Fig. 1$)$. MMP-2 tended to increase at 6 hour $(p=0.057)$ and was significantly elevated at 12 and 24 hours post exercise $(p<0.05$, Fig. 1). Neither, MMP-9 nor TSP-1 were significantly elevated at any time point up to 24 hours post exercise (Fig. 1).

\section{Study 2 - Temporal response of angiogenic regulators to exercise training. Age was} not different between the respective training groups. Body mass after training was approximately $5 \%$ greater in the 3 -day trained group $(p=0.04)$ compared to control mice, but not different in 5- and 7-day trained groups (Table 2). In contrast, body mass was $6 \%$ and $7 \%$ lower 
in 14- and 28-day trained mice, respectively $(p<0.01)$, compared to similar aged untrained control mice (Table 2). The absolute gastrocnemius muscle mass, as well as relative muscle mass normalized to total body mass (i.e. GA/BM), were not different among any of the groups. The same was observed for the plantaris (PLT) and soleus (SOL) muscles, with one exception, where SOL/BM tended to be greater $(p=0.04)$ in 28-day trained mice compared to controls (Table 2).

Training performance and muscle capillarity. Voluntary wheel running behavior was similar when comparing performance measures (i.e. average daily running speed, daily maximal running speed, and time spent running each day) on the same relative day of training, indicating similar training behavior and pattern between groups (data not shown). However, as would be expected, measures of exercise performance (i.e. average running speed and greater distance run each day) increased with increasing exposure to training (Table 3). Although time spent running also decreased with increasing days training $(p<0.05)$, the concommitant increased average running speed resulted in an overall effect of an increase in the average distance run each day. Maximal running speed performed during daily voluntary wheel training was not different between any of the trained groups (Table 3).

Training increased capillary-to-fiber ratio $(\mathrm{C}: \mathrm{F})$ in the gastrocnemius muscle of mice beginning with 7-days of training, and increased further with 14- and 28-days of voluntary wheel training ( $p<0.01$, at all time points) (Fig. 2).

Basal muscle protein expression following training. Shown in Figure 3, VEGF $(p=0.02)$ and MMP-2 ( $p=0.03)$ were significantly lower after 3 days of training compared to the control group. After 5 days of training, nucleolin $(p=0.03)$ was elevated and MMP-9 $(p=0.01)$ was reduced compared to the control group. The reduction in MMP-9 persisted with 7 and 14 days training $(p<0.01)$. MMP-2 was also elevated with 5 days training compared to 3 day trained 
levels, which also persisted with 7 and 14 days of training. In addition to altered MMP-2 and MMP-9 at 7 days of training, basal VEGF protein levels increased $(p=0.03)$. Following 14 days of training, VEGF levels were not different than basal control levels, but was significanly lower after 28 days of training $(p<0.01)$. TSP-1 in $14 d$ and $28 d$ trained mice was significantly greater compared to $5 d$ and $7 d$ trained mice $(p<0.05)$, but only tended to increase compared to basal control levels $(p<0.10)$.

Acute response to exercise following training. Following training, the angiogenic response to a single 1 -hour exercise bout (treadmill running at $20 \mathrm{~m} / \mathrm{min}, 10^{\circ}$ incline) was assessed at the 4-hour time point following the exercise bout. It is notable, of three angiogenic regulators found to respond to acute exercise in the (untrained) control mice (i.e. VEGF, endostatin, and nucleolin), none were seen to be responsive to acute exercise after 7-days of training (Fig. 3) when C:F was significantly increased (Fig. 2). But, the VEGF response to acute exercise was present with 3- and 5-days of training. Given that muscles were harvested at 4hours post exercise in each of the trained groups, the responses of angiogenic regulators (e.g. MMP-2, MMP-9 and TSP-1) whose peak responses occurred either before or after this time point would have limited, if any, interpretative value.

\section{Discussion}

The principal finding of this study is that the expression of key angioregulatory proteins are markedly up-regulated in either early (2-4 hours) or late phase (12-24 hours) post acute exercise. We find that VEGF, endostatin, and nucleolin protein levels were elevated in the early phase, whereas other regulators (such as MMP-2, and to lesser extent TSP-1) responded much later post exercise, showing that exercise elicits a complex but coordinated process involving positive and negative regulators. The new observation that nucleolin was increased with acute 
exercise is particularly interesting since it may interact and influence several key angiogenic mitogens (i.e. VEGF, MMP-9 and endostatin). Our data also provide evidence that chronic exercise training alters the responsiveness of VEGF to exercise after capillary adaptation has taken place. Collectively, these data provide evidence supporting the idea that exercise-induced skeletal muscle angiogenesis is a multifaceted processes involving positive and negative regulators and whose responses are temporally coordinated to limit or prevent angiogenesis in response to acute exercise, but which are differentially altered by chronic exercise in manner that allows or faciliates capillary expansion.

Temporal responses to single bout of acute exercise

The skeletal muscle VEGF mRNA response to exercise has been well characterized with peak responses occuring between 1-2 hours and returning to baseline levels by 4-8 hours (Breen et al., 1996; Gustafsson et al., 2005). Our data show that peak VEGF protein expression in mice occurs between 2-4 hours following a single bout of exercise, which is consistent with a transcriptionally-mediated event that contributes to the rise of VEGF protein expression (Tang et al., 2002). Nonetheless, we cannot exclude the possibility that exercise-induced posttranslational modifications may have also contributed to the rise in VEGF protein. The observed temporal response in this study, is also consistent with a previous report in rats showing significantly elevated VEGF protein 4-hours post exercise in the soleus and plantaris muscles (Malek et al., 2010). In contrast, VEGF protein levels measured from muscle biopsy in human vastus lateralis muscle have been reported to be lower immediately following exercise and return to baseline levels by 4 hours post exercise (Gavin et al., 2004). Likewise, another study reports no VEGF mRNA or protein response to acute exercise in young and old adults 4-hours after exercise (․ㅡan et al., 2006). While there are several studies that show a significant VEGF 
mRNA response to acute exercise in healthy adults (Gustafsson et al., 1999; $\underline{\text { Richardson et al., }}$ 1999; Gustafsson \& Sundberg, 2000; Richardson et al., 2000), none that have analyzed muscle biopsies have found elevated muscle VEGF protein between 0-6 hours post exercise. Thus, although the temporal response of exercise at the transcriptional (mRNA) level may be similar between rodents and humans, translation at the protein level appear to have very different temporal responses. At present there is not a clear explanation for this discrepancy at the protein level between rodents and humans, but one concern may be that the relatively small amount of muscle tissue that can feasibly be obtained from a human muscle biopsy may not fully represent the proteomic response occurring within the whole muscle.

Similar to VEGF, we observed that endostatin was significantly elevated in the gastrocnemius at 2- and 4-hours post exercise. Our data are consistent with the observation that circulating endostatin levels are elevated following acute exercise in humans ( $\underline{\mathrm{Gu}}$ et al., 2004; Rullman et al., 2007; Suhr et al., 2007). This response may seem counter intuitive, but it has been previously shown that acute exercise increases gene expression of other negative angiogenic regulators, such as TSP-1 (lfert et al., 2006). The prevailing concept is that up regulation of both positive and negative angiogenic regulators occurs in response to a single acute exercise bout to limit and/or prevent unnecessary utilization of energy and biological resources for capillary expansion in response to a single, one-time, stimulus. And that, capillary expansion only occurs in response to reoccurring or repeated exercise-induced mechanical or metabolic stress (Egginton, 2009; Olfert \& Birot, 2011). The response observed for endostatin in this study adds credence to this idea. With this in mind, we were surprised to observe no significant increase in muscle TSP-1 protein expression, particularly given the recent evidence supporting a role for TSP-1 as a key negative angiogenic regulator in skeletal muscle (Olfert et

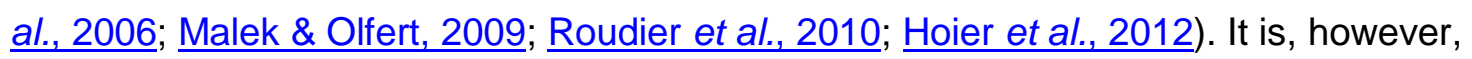
interesting to note that there was a trend for TSP-1 protein levels to increase at 12-24 hours 
post exercise $(p=0.09)$. Therefore, it may be that the peak TSP-1 response occurs beyond 24 hours, which was not measured in our study. Another recent study also found no change in TSP-1 protein levels in mice 4 hours following acute exercise (Audet et al., 2011), but in light of the present data this is not surprising. Based on these data, it might be interpreted that TSP-1 is unimportant in regulating exercise-induced skeletal muscle angiogenesis. But, given the robust TSP-1 mRNA response to exercise in rodents (lfert et al., 2006; Kivela et al., 2008) and humans (Hoier et al., 2012; Hoier et al., 2013), it may be premature to rule out the involvement of TSP-1. Further studies examining and documenting the TSP-1 protein responses to exercise are needed, before we may fully appreciate its contribution.

We also report the expression of nucleolin, which has been used as a marker of endothelial cell undergoing angiogenesis (hristian et al., 2003), and whose activity has been linked to endostatin (Shi et al., 2007), as well as interacting with VEGF and MMP-9 (Fahling et al., 2005; Huang et al., 2006). Given the increase in nucleolin at 2 and 4 hours post exercise, at a time point similar to the time profile seen with VEGF and endostatin, it is tempting to speculate that VEGF (in response to exercise) may help to facilitate translocation of nucleolin from the cell nucleus to the cell surface of proliferating endothelial cells (Christian et al., 2003; Huang et al.. 2006). And, if nucleolin serves as a binding partner for endostatin to be internalized on proliferating endothelial cells (Shi et al., 2007; Song et al., 2012), this would provide an effective means for feedback regulation that could limit and/or brake the angiogenic response of VEGF to an acute exercise stimulus. To our knowledge, no previous studies have investigated nucleolin in skeletal muscle in response to exercise, but based on this evidence we believe further investigation into nucleolin and its potential interaction with VEGF and endostatin are warranted.

The importance of MMPs, particularly MMP-9 and MMP-2, have been recognized in the context of muscle angioadaptation ( 2003; Milkiewicz \& Haas, 2005), but the temporal responses to acute exercise are still poorly 
defined. We found no significance increase in MMP-9 protein in response to acute exericse in our mice, but did observed a significant elevation during the late phase (12-24 hours) after exercsie. A recent report using the vastus lateralis muscle of exercising humans has reported no increase in MMP-2 protein following acute exercise (Rullman et al., 2007), but based on our data this is perhaps not surprising since those data were obtained 2-hours post exercise. Other studies have reported increases in circulating MMP-2 protein in humans 30-min after acute high-intensity exercise (Suhr et al., 2007) and up to 1-hour post exercise in endurance track athletes (Suhr et al., 2010). But it is presently not clear whether circulating levels of MMPs have a significant impact, or even a direct relationship, on skeletal muscle angiogenesis per se (Rullman et al., 2012). For MMP-9, we saw no significant skeletal muscle MMP-9 protein response following acute exercise in mice. But, in humans, Rullman et al. (2007) have reported an increase in MMP-9 mRNA and total protein in human vastus lateralis muscle immediately after and 2-hours post acute exercise. Likewise, Suhr et al. (Suhr et al., 2007) report increased circulating MMP-9 from 1-4 hours after exercise in track athletes. While some of the conflicting outcomes reported in skeletal muscle may be attributable to the varying time points selected for analysis post exercise, it may also be (as that seen for VEGF) that rodents and humans exhibit different temporal responses for MMPs in response to exercise. We also cannot rule out the possibility that some of the conflicting results may be explained by differing exercise intensity performed between studies (discussed further in following section).

\section{Temporal effects of training on muscle capillarity}

Skeletal muscle capillary adaptation to training has been well studied since it was first reported by Vanotti \& Magiday (1934); and as seen in Table 3, the voluntary wheel training paradigm we used resulted in significant increases in capillary-to-fiber ratio starting with 7-days 
of training, and continued to increase with longer exposure (i.e. 14- or 28-days) to daily exercise. While it was expected that animals with longer exposure to training would exhibit better exercise performance attributes (such as the ability to run longer and/or run at a faster average speed) compared to those with fewer training days (Table 3), it is important to note that we monitored daily training behaivor in all groups and found no difference in the daily absolute performance measures of mice across the training groups when compared at the same relative day in the training paradigm (i.e. comparing running metrics in all groups at 3-day, or at 5-day, etc). This means the responses between the trained groups we observed in our study are not likely due to differences in volunatry training behavior, but rather most likely to the level of physiological adaptations that occurred with training (e.g. level of muscle capillarity). Given the plieotrophic effects of whole body exercise, we cannot directly point to any single traininginduced physiological adaptations (such as increased muscle capillarity) as being solely responsible for altering the responses we observed, but it is reasonable to interpret the individual and collective outcome of these angiogenic regulators with respect to their putative affect on angiogenesis.

\section{Basal response of angiogenic regulators following exercise training}

Basal expression of VEGF protein was lower in 3- and 28-day trained mice, but elevated at 7-days training, compared to (untrained) controls. The lower basal VEGF protein levels after 3-days of training was surprising, and directly contrasts to a previous report in the plantaris muscle of healthy mice (Waters et al., 2004). The reason for this discrepancy is not clear, but the rise in basal VEGF after 7-days of training, which coincides with elevated muscle capillarity (seen in Fig. 2) is consistent with the existing data (Waters et al., 2004), and suggests a basal condition that is favorable for angiogenesis. Surprisingly, we observed that basal VEGF levels 
fell in 28-day trained mice compared to basal untrained levels. This might suggest a feedback mechanism designed to limit or slow down capillary expansion once a sufficient level of angiogenesis has taken place. The observation of normalized muscle basal VEGF protein expression following training, after capillary expansion has occurred, has also been observed in humans following training (Hoier et al., 2012). Taken together, our temporal analysis of basal VEGF in association with training-induced muscle capillary adaptation provide evidence of a complex control of VEGF, and suggest that basal level expression may be linked to the degree of capillary adaptation. This idea is supported by microarray analysis indicating that the genomic responses to aerobic exercise in human skeletal muscle is influenced by the magnitude of physiological adaptation which has occurred (Timmons et al., 2005), which emphasizes that comparing and contrasting VEGF responses (or perhaps of any angioregulatory factor) to training among different studies may be difficult to interpret unless the level of capillary adaptation is also known and reported.

With respect to TSP-1, we found that basal protein levels of this anti-angiogenic factor were decreased with 5- and 7-days training compared to 14- and 28-days (Fig. 3), suggesting that longer exposure to training may act to slow the capillary expansion by elevating basal TSP1 levels. This is consistent with the notion that a balance between positive and negative angiogenic regulators control skeletal muscle angiogenesis, and is supported by our data showing reduced basal VEGF, but elevated basal TSP-1, in 28-day trained mice. This is also consistent with mRNA expression profile which have been reported separately for TSP-1 (Olfert et al., 2006) and VEGF (Gavin \& Wagner, 2001) with short-term training (up to 5-days) in the skeletal muscle of rats. In contrast, however, Hoier et al. have recently shown unaltered basal TSP-1 and VEGF proteins following 4-weeks of bicycle ergometer training that resulted in an increase of muscle C:F (Hoier et al., 2012). There could be several explanations for the apparent difference. First, as we have previously noted with respect to acute exercise 
responses, there could simply be differences in the temporal response of humans and rodents to exercise. Another potential explanation may be that myocytes are not the source of TSP-1. Indeed, TSP-1 is an extracellular matrix protein that is produced by a wide variety of cells, such as platelets, fibroblasts, macrophages, and other cells (Bornstein, 1992); thus exercise-induced alterations in TSP-1 could originate from non-myocyte sources. Supporting this, Hoier et al. (2013) have recently shown decreased TSP-1 protein in extracellular muscle dialysate fluid (obtained from indwelling muscle catheters) after 4-weeks moderate-to-high-intensity training in man. On the other hand, in the same report, Hoier et al. (2013) show increased TSP-1 protein in muscle dialysate following high-intensity intermittent exercise in the same subjects, emphasizing that exercise intensity (and subsequent physiological adaptation) likely exerts an important influence on the angioregulatory protein response of skeletal muscle to training. Thus, it may be difficult to compare training responses unless the exercise intensity is similar, and that it may be even more difficult to compare across species (as we have previously seen for VEGF between rodents and humans).

There are a limited number of studies examining the involvement of endostatin in exercise-induced skeletal muscle angiogenesis. While circulating endostatin levels under basal conditions have been shown to be reduced after six months of training in untrained men and short-/long-track athletes (Brixius et al., 2008; Suhr et al., 2010), our data examining basal muscle expression of endostatin do not show this in mice. Based on the human data, it could be that we needed a longer training period to get a reduction in endostatin, but given the significant rise in muscle capillarity observed after 28-days of training it would appear that a reduction in basal endostatin was not needed for capillary expansion to occur. However, based on the acute protein response we, and others, have observed for endostatin, it appears that endostatin may be one of several potential negative regulators that serve to prevent or limit angiogenesis in response to acute exercise. 
As seen with endostatin, no significant changes in basal protein expression of nucleolin were observed with training. If the role of nucleolin involves interaction with endostatin in response to acute exercise, then this is perhaps not surprising. However, before the importance of nucleolin can be determined in response to acute exercise or training, additional studies examining the effect of exercise on the translocation of nucleolin to the endothelial cell surface, and to what extent VEGF and endostatin are dependent on nucleolin are needed.

Because previous reports show increased skeletal muscle MMP mRNA and protein in response to electrically-stimulated contractions and/or prazosin-mediated increases in vessel shear stress (haas et al., 2000; Rivilis et al., 2002; Brown \& Hudlicka, 2003), we were surprised to observe that basal MMP-2, and to a larger extent MMP-9, protein expression were significantly lower with training in our mice (Fig. 3). Our data are in stark contrast to existing studies, most of which show elevated MMP levels following various intensity and length of training in rats (ㅂaas et al., 2000; Carmeli et al., 2007) and humans (ullman et al., 2009). For example, single-leg training in humans for only 10 days is reported to increase both MMP-2 and MMP-9 mRNA and total protein activity in the exercising muscle (ullman et al., 2009). An explanation for the discrepancies between our data and those previously published may be due to training paradigm (i.e. voluntary wheel running) we used. Using a rat model, Carmeli et al. (2005) have reported that MMP-2 response to training is exercise intensity dependent, such that high intensity training ( $70 \%$ of maximal oxygen consumption, VO2max) was needed to increase MMP-2 expression in the gastrocnemius muscle of rats, whereas training at $~ 50 \%$ of VO2max was not sufficient (Carmeli et al., 2005). Despite the high daily volume of training voluntarily performed by our mice (up to $6 \mathrm{~km}$ per day), the average running speed during training ranged between 12 to $18 \mathrm{~m} / \mathrm{min}(0.71-1.06 \mathrm{~km} / \mathrm{hr}$, Table 3$)$. If one accepts the maximal treadmill running speed for C57BI/6 mice is between 35-45 m/min (ㅂøydal et al., 2007; Malek \& Olfert, 2009; Olfert et al., 2009), the average running speed during voluntary wheel 
training performed by our mice would only be approximately $34-40 \%$ of their maximum exercise capacity. Thus it may be that we do not find significant increases in MMP due to the lowintensity training that was voluntarily performed by our mice. Nonetheless, it should be noted that we did find that 5-, 7- and 14-days of training resulted in significant increase in total basal MMP-2 protein compared to that seen with 3-days training (Fig. 3), and that a similar trend existed for elevated basal MMP-2 with 7 days training compared to basal levels in control mice ( $p=0.07$, Fig. 3). Thus, exercise intensity may play an important role in MMP response to exercise training. This could, in part, also explain the discrepancies in our acute exercise data (showing a non-significant response, Fig. 1) compared to the increase in MMP-9 protein seen in humans immediately and 2-hours after a single acute bout of exercise (Rullman et al., 2012). We estimate the relative intensity of the single 1-hour exercise bout (at $20 \mathrm{~m} / \mathrm{min}$ with 10-degree incline) performed by our untrained mice is about $50-60 \%$ of the maximal capacity in these mice. Therefore, even though it appears there was a tendency for MMP-9 to be elevated at 0and 2-hours post acute exercise (compared to controls) (Fig. 1), the exercise intensity performed for acute exercise might explain why a greater response was not observed.

Acute exercise responses of angiogenic regulators following exercise training

To examine that acute responsiveness of these angioregulatory proteins in trained mice, we subjected mice in each of the trained groups to a 1-hour standardized treadmill exercsie bout (i.e. $20 \mathrm{~m} / \mathrm{min}$, 10-degree incline) after a 24-hour window of time following the removal of the running wheel from the animals cage. We selected the 4-hour time-point post exercise to assess the responsivness to acute exercise, because it corresponded to the most significant time point we initially observed in the untrained mice (Study 1, Fig. 1). Accordingly, it must be recognized that we are only able to interpret the outcomes to acute exercise for VEGF, 
endostatin, and nucleolin, because the responses for these factors occurred at this time point. Nevertheless, due to scarcity of data and an expanding need to document the molecular interplay that occurs in trained versus sedentary conditions, we have still reported (in Fig. 3) the outcome for acute exercise for all the factors we evaluated, even though the interpretative value of TSP-1, MMP-2 and MMP-9 (which were found not to respond at 4 hours) are likely to be less informative. As such, we have limited our discussion here to focus only on those factors which were significant 4-hours post acute exercise (i.e. VEGF, endostatin and nucleolin).

As reported in previous studies, skeletal muscle VEGF protein was significantly increased in response to acute exercise in untrained subjects (Malek et al., 2010; $\underline{\text { Hoier et al., }}$ 2012). Our study reveals that the VEGF response is lost after 7-days of training, and remains attenuated following 14- and 28-days of training in mice (Fig. 3). The fact that these time points correspond temporally to a significant increase in muscle capillarity (Fig. 2) would suggest that elevated muscle capillarity (and/or other training related adaptations) have likely reduced or lessened metabolic stress (e.g. shear stress, local tissue hypoxia, stretch, etc) associated with exercise, and therefore reduced the underlying stimulus for activating VEGF. This idea is supported by previous data showing that in rats trained in hypoxia (which resulted in increased muscle capillarization) the VEGF mRNA response to exercise was blunted, whereas rats trained at the same absolute training intensity in normoxia (which did not increase muscle capillary, presumably due to the significantly lower relative training intensity they experienced) exhibited a similar VEGF mRNA response to acute exercise as that seen in untrained rats (Olfert et al., 2001). Likewise, neither endostatin nor nucleolin were found to exhibit a significant response to acute exercise following training (Fig. 3). Given the basal levels of endostatin and nucleolin are unchanged with training, the most likely interpretation of these data are the that endostatin and nucleolin only play a significant role in regulating the initial muscle response to exercise by 
limiting and/or preventing angiogenesis in response to acute exercise, and not in response to chronic exercise training.

In conclusion, the principal finding of this study is that angiognesis resulting from exercise training is dependent on coordination of both positive (VEGF, MMP-2, nucleolin) and negative (TSP-1, endostatin) angiogenic regulators. In response to acute exercise there appears to be early (2-4 hours) and late (12-24 hours) phase protein responses, whereas with training these responses are attenuated or lost. To our knowledge, we believe these are the first data to suggest that nucleolin may serve (in concert with VEGF and endostatin) as an important peptide in response to acute exercise, such that endostatin may serve to inhibit the initial angiogenic response to acute exercise by countering the actions of VEGF via a nucleolinmediated mechanism. These data also highlight that the temporal responses we observed in mice, particularly for VEGF, MMP-2 and MMP-9, may not be directly comparable to humans. Nonetheless, when taken together, our results show that there is a complex coordination in the protein responses of both positive and negative angiogenic factors that correspond with traininginduced muscle capillary adaptation. 


\section{Acknowledgements}

Funding support was provided, in part by, West Virginia University Research Funding and Development Grant (Olfert), American Heart Association 10BGIA3630002 (Olfert), Tobacco Related Disease Research Program \# 14KT-0091 (Olfert), and Cornelius Hopper TRDRP Supplement Award (Gutierrez-Reed).

Authors have no conflict of interest to disclose. 


\section{References}

Audet GN, Meek TH, Garland Jr T \& Olfert IM (2011). Expression of angiogenic regulators and skeletal muscle capillarity in selectively bred high aerobic capacity mice. Experimental Physiology 96, 1138-1150.

Bloor CM (2005). Angiogenesis during exercise and training. Angiogenesis 8, 263-271.

Bornstein $P$ (1992). Thrombospondins: structure and regulation of expression [published erratum appears in FASEB J 1993 Jan;7(1):237]. FASEB J 6, 3290-3299.

Breen EC, Johnson EC, Wagner H, Tseng HM, Sung LA \& Wagner PD (1996). Angiogenic growth factor mRNA responses in muscle to a single bout of exercise. J Appl Physiol 81, 355361.

Brixius K, Schoenberger S, Ladage D, Knigge H, Falkowski G, Hellmich M, Graf C, Latsch J, Montie GL, Prede GL \& Bloch W (2008). Long-term endurance exercise decreases antiangiogenic endostatin signalling in overweight men aged 50-60 years. $\mathrm{Br} \mathrm{J}$ Sports Med 42 , 126-129;.

Brown MD \& Hudlicka O (2003). Modulation of physiological angiogenesis in skeletal muscle by mechanical forces: involvement of VEGF and metalloproteinases. Angiogenesis 6, 1-14.

Carmeli E, Haimovitz T \& Nemcovsky EC (2007). Cathepsin D and MMP-9 activity increase following a high intensity exercise in hind limb muscles of young rats. J Basic Clin Physiol Pharmacol 18, 79-86.

Carmeli E, Moas M, Lennon S \& Powers SK (2005). High intensity exercise increases expression of matrix metalloproteinases in fast skeletal muscle fibres. Exp Physiol 90, 613-619.

Christian S, Pilch J, Akerman ME, Porkka K, Laakkonen P \& Ruoslahti E (2003). Nucleolin expressed at the cell surface is a marker of endothelial cells in angiogenic blood vessels. J Cell Biol 163, 871-878.

Egginton S (2009). Invited review: activity-induced angiogenesis. Pflugers Arch 457, 963-977.

Fahling M, Steege A, Perlewitz A, Nafz B, Mrowka R, Persson PB \& Thiele BJ (2005). Role of nucleolin in posttranscriptional control of MMP-9 expression. Biochim Biophys Acta 1731, 32-40.

Gavin TP, Robinson CB, Yeager RC, England JA, Nifong LW \& Hickner RC (2004). Angiogenic growth factor response to acute systemic exercise in human skeletal muscle. J Appl Physiol 96, 19-24.

Gavin TP \& Wagner PD (2001). Effect of short-term exercise training on angiogenic growth factor gene responses in rat. J Appl Physiol 90, 1219-1226.

Gu J-W, Gadonski G, Wang J, Makey I \& Adair T (2004). Exercise increases endostatin in circulation of healthy volunteers. BMC Physiology 4, 2. 
Gustafsson T, Ameln H, Fischer H, Sundberg CJ, Timmons JA \& Jansson E (2005). VEGF-A splice variants and related receptor expression in human skeletal muscle following submaximal exercise. J Appl Physiol 98, 2137-2146.

Gustafsson T, Puntschart A, Kaijser L, Jansson E \& Sundberg CJ (1999). Exercise-induced expression of angiogenesis-related transcription and growth factors in human skeletal muscle. Am J Physiol 276, H679-685.

Gustafsson T \& Sundberg CJ (2000). Expression of angiogenic growth factors in human skeletal muscle in response to a singular bout of exercise. Am J Physiol Heart Circ Physiol 279, H31443145.

Haas TL, Milkiewicz M, Davis SJ, Zhou AL, Egginton S, Brown MD, Madri JA \& Hudlicka O (2000). Matrix metalloproteinase activity is required for activity-induced angiogenesis in rat skeletal muscle. Am J Physiol Heart Circ Physiol 279, H1540-1547.

Hoier B, Nordsborg N, Andersen S, Jensen L, Nybo L, Bangsbo J \& Hellsten Y (2012). Pro- and anti-angiogenic factors in human skeletal muscle in response to acute exercise and training. $\mathrm{J}$ Physiol 590, 595-606.

Hoier B, Passos M, Bangsbo J \& Hellsten Y (2013). Intense intermittent exercise provides weak stimulus for vascular endothelial growth factor secretion and capillary growth in skeletal muscle. Exp Physiol 98, 585-597.

Howlett RA, Kirkton SD, Gonzalez NC, Wagner HE, Britton SL, Koch LG \& Wagner PD (2009). Peripheral oxygen transport and utilization in rats following continued selective breeding for endurance running capacity. J Appl Physiol 106, 1819-1825.

Høydal MA, Wisløff U, Kemi OJ \& Ellingsen $\varnothing$ (2007). Running speed and maximal oxygen uptake in rats and mice: practical implications for exercise training. European Journal of Cardiovascular Prevention \& Rehabilitation 14, 753-760.

Huang Y, Shi H, Zhou H, Song X, Yuan S \& Luo Y (2006). The angiogenic function of nucleolin is mediated by vascular endothelial growth factor and nonmuscle myosin. Blood 107, 35643571.

Hudlicka O, Brown M \& Egginton S (1992). Angiogenesis in skeletal and cardiac muscle. Physiol Rev 72, 369-417.

Kivela R, Silvennoinen M, Lehti M, Jalava S, Vihko V \& Kainulainen H (2008). Exercise-induced expression of angiogenic growth factors in skeletal muscle and in capillaries of healthy and diabetic mice. Cardiovasc Diabetol 7, 13.

Lloyd PG, Prior BM, Li H, Yang HT \& Terjung RL (2005). VEGF receptor antagonism blocks arteriogenesis, but only partially inhibits angiogenesis, in skeletal muscle of exercise-trained rats. Am J Physiol Heart Circ Physiol 288, H759-768.

Lloyd PG, Prior BM, Yang HT \& Terjung RL (2003). Angiogenic growth factor expression in rat skeletal muscle in response to exercise training. Am J Physiol Heart Circ Physiol 284, H16681678. 
Malek MH \& Olfert IM (2009). Global deletion of thrombospondin-1 increases cardiac and skeletal muscle capillarity and exercise capacity. Exp Physiol 94, 749-760.

Malek MH, Olfert IM \& Esposito F (2010). Detraining losses of skeletal muscle capillarization are associated with vascular endothelial growth factor protein expression in rats. Exp Physiol 95, 359-368.

Mathieu-Costello O \& Hepple RT (2002). Muscle structural capacity for oxygen flux from capillary to fiber mitochondria. Exerc Sport Sci Rev 30, 80-84.

Milkiewicz M \& Haas TL (2005). Effect of mechanical stretch on HIF-1\{alpha\} and MMP-2 expression in capillaries isolated from overloaded skeletal muscles: laser capture microdissection study. Am J Physiol Heart Circ Physiol 289, H1315-1320.

Mrazkova O, Grim M \& Carlson BM (1986). Enzymatic heterogeneity of the capillary bed of rat skeletal muscles. Am J Anat 177, 141-148.

Olfert IM \& Birot O (2011). Importance of Anti-angiogenic Factors in the Regulation of Skeletal Muscle Angiogenesis. Microcirculation 18, 316-330.

Olfert IM, Breen EC, Gavin TP \& Wagner PD (2006). Temporal thrombospondin-1 mRNA response in skeletal msucle exposed to acute and chronic exercise. Growth Factors 24, 253259.

Olfert IM, Breen EC, Mathieu-Costello O \& Wagner PD (2001). Skeletal muscle capillarity and angiogenic mRNA levels after exercise training in normoxia and chronic hypoxia. J Appl Physiol 91, 1176-1184.

Olfert IM, Howlett RA, Tang K, Dalton ND, Gu Y, Peterson KL, Wagner PD \& Breen EC (2009). Muscle-specific VEGF deficiency greatly reduces exercise endurance in mice. J Physiol 578, 1755-1767.

Olfert IM, Howlett RA, Wagner PD \& Breen EC (2010). Myocyte vascular endothelial growth factor is required for exercise-induced skeletal muscle angiogenesis. Am J Physiol Regul Integr Comp Physiol 299, R1059-1067.

Prior BM, Yang HT \& Terjung RL (2004). What makes vessels grow with exercise training? J Appl Physiol 97, 1119-1128.

Richardson RS, Wagner H, Mudaliar SR, Saucedo E, Henry R \& Wagner PD (2000). Exercise adaptation attenuates VEGF gene expression in human skeletal muscle. Am J Physiol Heart Circ Physiol 279, H772-778.

Richardson RS, Wagner H, Mudaliar SRD, Henry R, Noyszewski EA \& Wagner PD (1999). Human VEGF gene expression in skeletal muscle: effect of acute normoxic and hypoxic exercise. Am J Physiol 277, H2247-H2252.

Rivilis I, Milkiewicz M, Boyd P, Goldstein J, Brown MD, Egginton S, Hansen FM, Hudlicka O \& Haas TL (2002). Differential involvement of MMP-2 and VEGF during muscle stretch- versus shear stress-induced angiogenesis. Am J Physiol Heart Circ Physiol 283, H1430-1438. 
Roudier E, Gineste C, Wazna A, Dehghan K, Desplanches D \& Birot O (2010). Angioadaptation in unloaded skeletal muscle: new insights into an early and muscle type-specific dynamic process. J Physiol 588, 4579-4591.

Rullman E, Norrbom J, Stromberg A, Wagsater D, Rundqvist H, Haas T \& Gustafsson T (2009). Endurance exercise activates matrix metalloproteinases in human skeletal muscle. J Appl Physiol 106, 804-812.

Rullman E, Olsson K, Wagsater D \& Gustafsson T (2012). Circulating MMP-9 during exercise in humans. Eur J Appl Physiol [Epub ahead of print] Nov 17, 2012.

Rullman E, Rundqvist H, Wagsater D, Fischer H, Eriksson P, Sundberg CJ, Jansson E \& Gustafsson T (2007). A single bout of exercise activates matrix metalloproteinase in human skeletal muscle. J Appl Physiol 102, 2346-2351.

Ryan NA, Zwetsloot KA, Westerkamp LM, Hickner RC, Pofahl WE \& Gavin TP (2006). Lower skeletal muscle capillarization and VEGF expression in aged vs. young men. J Appl Physiol $100,178-185$.

Shi H, Huang Y, Zhou H, Song X, Yuan S, Fu Y \& Luo Y (2007). Nucleolin is a receptor that mediates antiangiogenic and antitumor activity of endostatin. Blood 110, 2899-2906.

Snyder GK (1987). Muscle capillarity in chicks following hypoxia. Comp Biochem Physiol 87A, 819-822.

Snyder GK, Farrelly C \& Coelho JR (1992). Adaptations in skeletal muscle capillarity following changes in oxygen supply and changes in oxygen demands. Eur J Appl Physiol 65, 158-163.

Song N, Ding Y, Zhuo W, He T, Fu Z, Chen Y, Song X, Fu Y \& Luo Y (2012). The nuclear translocation of endostatin is mediated by its receptor nucleolin in endothelial cells. Angiogenesis 15, 697-711.

Srivastava M \& Pollard HB (1999). Molecular dissection of nucleolin's role in growth and cell proliferation: new insights. Faseb J 13, 1911-1922.

Suhr F, Brixius K, de Marees M, Bolck B, Kleinoder H, Achtzehn S, Bloch W \& Mester J (2007). Effects of short-term vibration and hypoxia during high-intensity cycling exercise on circulating levels of angiogenic regulators in humans. J Appl Physiol 103, 474-483.

Suhr F, Rosenwick C, Vassiliadis A, Bloch W \& Brixius K (2010). Regulation of extracellular matrix compounds involved in angiogenic processes in short- and long-track elite runners. Scand J Med Sci Sports 20, 441-448.

Tang K, Breen EC \& Wagner PD (2002). Hu protein R-mediated posttranscriptional regulation of VEGF expression in rat gastrocnemius muscle. Am J Physiol Heart Circ Physiol 283, H14971504.

Timmons J, Jansson E, Fischer H, Gustafsson T, Greenhaff P, Ridden J, Rachman J \& Sundberg $C$ (2005). Modulation of extracellular matrix genes reflects the magnitude of physiological adaptation to aerobic exercise training in humans. BMC Biology 3, 19. 
Vanotti A \& Magiday M (1934). Untersuchungen zum Studium des Trainiertseins. Arbeitsphysiol 7, 615-622.

Wagner PD (1996). Determinants of maximal oxygen transport and utilization. Annu Rev Physiol $58,21-50$.

Waters RE, Rotevatn S, Li P, Annex BH \& Yan Z (2004). Voluntary running induces fiber typespecific angiogenesis in mouse skeletal muscle. Am J Physiol Cell Physiol 287, C1342-1348.

Williams JL, Cartland D, Rudge JS \& Egginton S (2006). VEGF trap abolishes shear stress- and overload-dependent angiogenesis in skeletal muscle. Microcirculation 13, 499-509. 
Blank Page 
TABLES AND FIGURES

\begin{tabular}{|c|c|c|c|c|c|c|}
\hline Group & Age (days) & Body Mass (g) & $\mathrm{GA}(\mathrm{mg})$ & PLT (mg) & SOL (mg) & HRT (mg) \\
\hline Controls & $72 \pm 0$ & $25.7 \pm 0.7$ & $137.0 \pm 3.2$ & $20.3 \pm 0.9$ & $6.4 \pm 0.4$ & $132.7 \pm 3.6$ \\
\hline 0 Hours & $71 \pm 0$ & $26.5 \pm 0.3$ & $146.9 \pm 2.7$ & $19.1 \pm 0.4$ & $7.4 \pm 0.5$ & $117.9 \pm 4.8$ \\
\hline 2 Hours & $72 \pm 0$ & $26.6 \pm 0.3$ & $139.0 \pm 4.5$ & $21.2 \pm 1.0$ & $8.2 \pm 0.3^{*}$ & $133.4 \pm 4.2$ \\
\hline 4 Hours & $71 \pm 0$ & $23.6 \pm 0.7$ & $127.4 \pm 4.8$ & $18.2 \pm 1.4$ & $6.9 \pm 0.3$ & $130.3 \pm 4.6$ \\
\hline 6 Hours & $72 \pm 0$ & $24.4 \pm 0.4$ & $131.6 \pm 4.4$ & $18.8 \pm 0.3$ & $6.6 \pm 0.3$ & $123.5 \pm 4.6$ \\
\hline 12 Hours & $71 \pm 0$ & $25.3 \pm 0.2$ & $136.7 \pm 2.6$ & $21.1 \pm 1.6$ & $6.5 \pm 0.4$ & $117.6 \pm 2.4$ \\
\hline 24 Hours & $72 \pm 0$ & $25.2 \pm 0.4$ & $131.2 \pm 1.0$ & $19.2 \pm 0.4$ & $7.5 \pm 0.2$ & $128.4 \pm 2.5$ \\
\hline
\end{tabular}

Table 1. Mean $\pm S E$. Average age, body and muscle mass in mice sacrifice at the select times following a single acute exercise bout ( $n=4$ /group). GA=gastrocnemius, PLT=plantaris, SOL=soleus, HRT=heart. No significant differences are seen between mice in any of the groups. 


\begin{tabular}{|c|c|c|c|c|c|c|c|c|}
\hline & Age & Body Mass (g) & $\mathrm{GA}(\mathrm{mg})$ & GA/BM (\%) & PLT (mg) & PLT/BM (\%) & SOL (mg) & SOL/BM (\%) \\
\hline Control & $74 \pm 1$ & $25.0 \pm 0.4$ & $133 \pm 4$ & $0.53 \pm 0.01$ & $15.3 \pm 0.7$ & $0.061 \pm 0.003$ & $6.7 \pm 0.2$ & $0.027 \pm 0.001$ \\
\hline $3 d$ & $73 \pm 1$ & $26.3 \pm 0.4^{*}$ & $139 \pm 2$ & $0.53 \pm 0.01$ & $14.7 \pm 0.6$ & $0.056 \pm 0.002$ & $7.2 \pm 0.4$ & $0.027 \pm 0.001$ \\
\hline $5 d$ & $67 \pm 2$ & $24.4 \pm 0.5$ & $130 \pm 5$ & $0.53 \pm 0.01$ & $13.8 \pm 0.8$ & $0.057 \pm 0.004$ & $7.2 \pm 0.4$ & $0.029 \pm 0.002$ \\
\hline $7 d$ & $73 \pm 3$ & $25.1 \pm 0.3$ & $137 \pm 3$ & $0.55 \pm 0.01$ & $14.1 \pm 1.0$ & $0.056 \pm 0.004$ & $7.3 \pm 0.3$ & $0.029 \pm 0.001$ \\
\hline 14d & $81 \pm 4$ & $23.6 \pm 0.4^{*}$ & $123 \pm 4$ & $0.52 \pm 0.02$ & $17.2 \pm 1.2$ & $0.073 \pm 0.005$ & $7.3 \pm 0.7$ & $0.031 \pm 0.003$ \\
\hline 28d & $74 \pm 1$ & $23.3 \pm 0.5^{*}$ & $126 \pm 7$ & $0.54 \pm 0.02$ & $13.8 \pm 0.5$ & $0.059 \pm 0.002$ & $7.4 \pm 0.3$ & $0.032 \pm 0.001^{*}$ \\
\hline
\end{tabular}

Table 2. Mean \pm SE. Average age, body and muscle mass following voluntary wheel running training for $3,5,7,14$ or 28 (d)ays ( $\mathrm{n}=12$ /group). $\mathrm{BM}=$ body mass, $\mathrm{GA}=$ gastrocnemius, $\mathrm{PLT}=$ plantaris, $\mathrm{SOL}=$ soleus. ${ }^{*} p<0.05$. 


\begin{tabular}{|c|ccc|ccccc|cccc|}
\hline Group & $\begin{array}{c}\text { Average Time } \\
\text { Running (min) }\end{array}$ & $\begin{array}{c}\text { Average } \\
\text { Running Speed } \\
\mathbf{( k m} / \mathbf{h r})\end{array}$ & $\begin{array}{c}\text { Average } \\
\text { Distance Run } \\
\mathbf{( k m / d a y )}\end{array}$ & $\begin{array}{c}\text { Average Max } \\
\text { Speed (km/hr) }\end{array}$ \\
\hline $\begin{array}{c}\text { Start of } \\
\text { Training }\end{array}$ & 363 & \pm & 21 & 0.71 & \pm & 0.02 & 3.64 & \pm & 0.20 & 2.59 & \pm & 0.06 \\
\hline $\mathbf{3 d}$ & 319 & \pm & 27 & 0.75 & \pm & 0.02 & 4.19 & \pm & 0.39 & 2.63 & \pm & 0.06 \\
\hline $\mathbf{5 d}$ & 261 & \pm & $13^{*}$ & 0.85 & \pm & $0.04^{* *}$ & 4.05 & \pm & 0.34 & 2.64 & \pm & 0.04 \\
\hline $\mathbf{7 d}$ & 289 & \pm & 29 & 0.93 & \pm & $0.05^{* *}$ & 4.89 & \pm & $0.57^{*}$ & 2.54 & \pm & 0.07 \\
\hline $\mathbf{1 4 d}$ & 205 & \pm & $15^{* *}$ & 1.02 & \pm & $0.04^{* *}$ & 3.91 & \pm & 0.38 & 2.67 & \pm & 0.07 \\
\hline $\mathbf{2 8 d}$ & 247 & \pm & $19^{* *}$ & 1.06 & \pm & $0.05^{* *}$ & 5.26 & \pm & $0.59^{* *}$ & 2.72 & \pm & 0.05 \\
\hline
\end{tabular}

Table 3. Mean $\pm S E$. Characterization of voluntary running wheel training behavior following 3,5 , 7,14 or 28 (d)ays ( $n=12 /$ group). Data represented are average data obtained from the last 24 hours for each respective group, except for "Start of Training" which is the combined average for all groups after $1^{\text {st }}$ full day access to running $(n=60) .{ }^{*} p<0.01,{ }^{* *} p<0.005$. 


\section{Figure Legend}

Figure 1. Protein time course expression of VEGF, MMP-2, MMP-9, TSP-1 (165kDa), endostatin (22kDa) and nucleolin (100kDa) following single bout of acute exercise measured by ELISA or Western Blot analysis. Representative blots are shown. Control mice did not exercise, all others ran for 1 hour (at $20 \mathrm{~m} / \mathrm{min}, 10^{\circ}$ incline) and were sacrificed immediately (0), 2, 4, 6, 12 or 24 hours post exercise ( $n=4$ mice each group). A.U.= arbitary densitometry units respresenting Western blot analysis for which each respective factor has been normalized to the housekeeping gene GAPDH (40kDa) and/or $\beta$-tubulin $(55 \mathrm{kDa})$. * Significantly different compared to Basal Control group, $p<0.05$; \$ signifies $p \leq 0.10$ compared to Basal control.

Figure 2. Gastrocnemuis muscle capillary-to fiber ratio $(\mathrm{C}: \mathrm{F})$ in control, untrained, mice and in mice that voluntarily trained for $3,5,7,14$ or 28 (d)ays ( $n=6 /$ group). ${ }^{* *} p<0.01$ compared to control.

Figure 3. Time course protein expression of VEGF, MMP-2, MMP-9, nucleolin (100kDa), endostatin (22kDa) and TSP-1 (165kDa) following training measured by ELISA or Western Blot analysis. Representative blots are shown. Control mice are untrained, all others voluntarily trained using a running wheel for $3,5,7,14$, or 28 days ( $n=12 /$ group). Following training, half of the mice per group $(n=6)$ were elevated under basal conditions, and the other half $(n=6)$ performed a single bout of exercise ( 1 hour, $20 \mathrm{~m} / \mathrm{min}, 10^{\circ}$ incline) with muscle harvested 4hours post exercise. A.U.= arbitary densitometry units respresenting Western blot analysis for which each respective factor has been normalized to the housekeeping gene GAPDH (40kDa). * significantly different compared to Basal Control group, $p<0.05$; $\ddagger$ significantly different between Basal and Acute exercise within same training group, $p<0.05$; $\uparrow$ significantly different compared to 3 day trained Basal levels, $p<0.05$; \# significantly different compared to 14-day trained Basal levels, $p<0.05 ; \$$ signifies $p \leq 0.10$ compared to Basal control. 

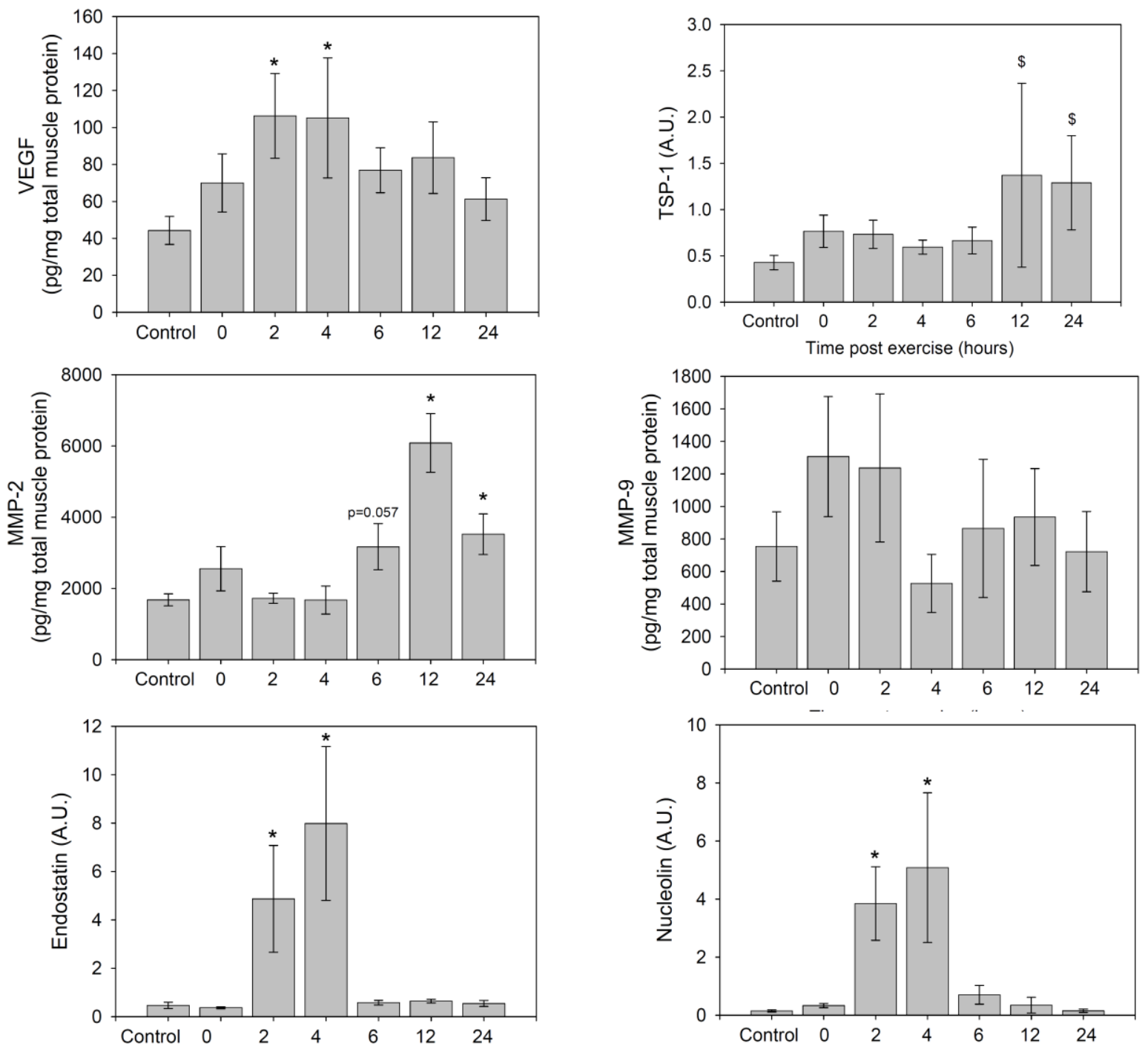

Nucleolin Endostatin GAPDH

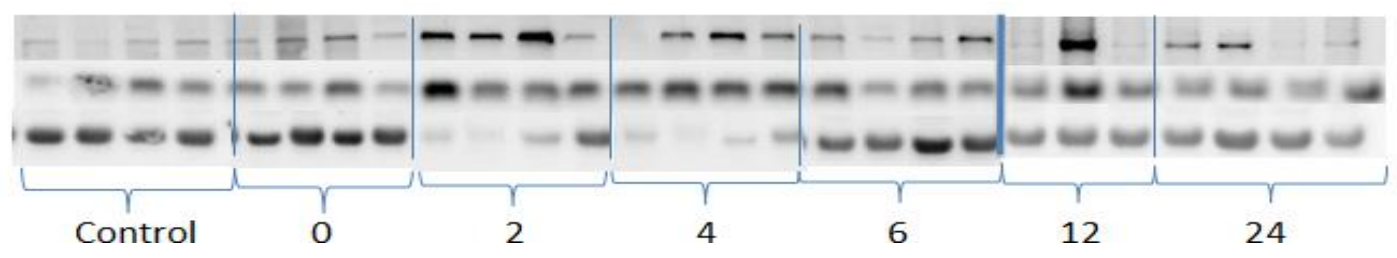

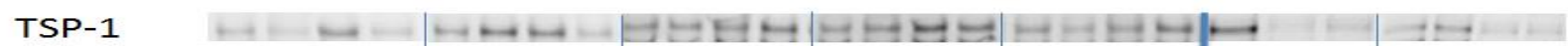
B-tubulin

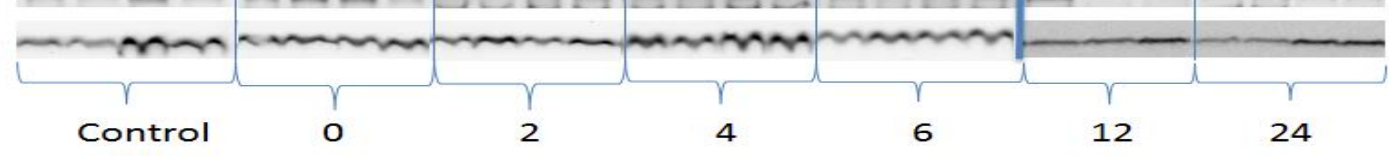

Figure 1. 


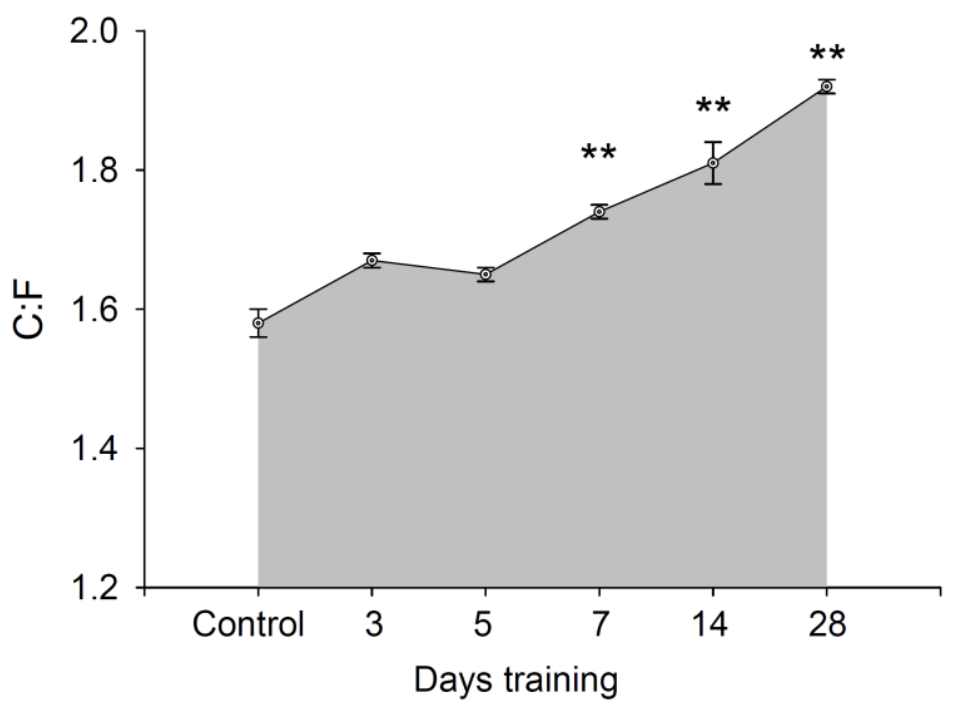

Figure 2. 

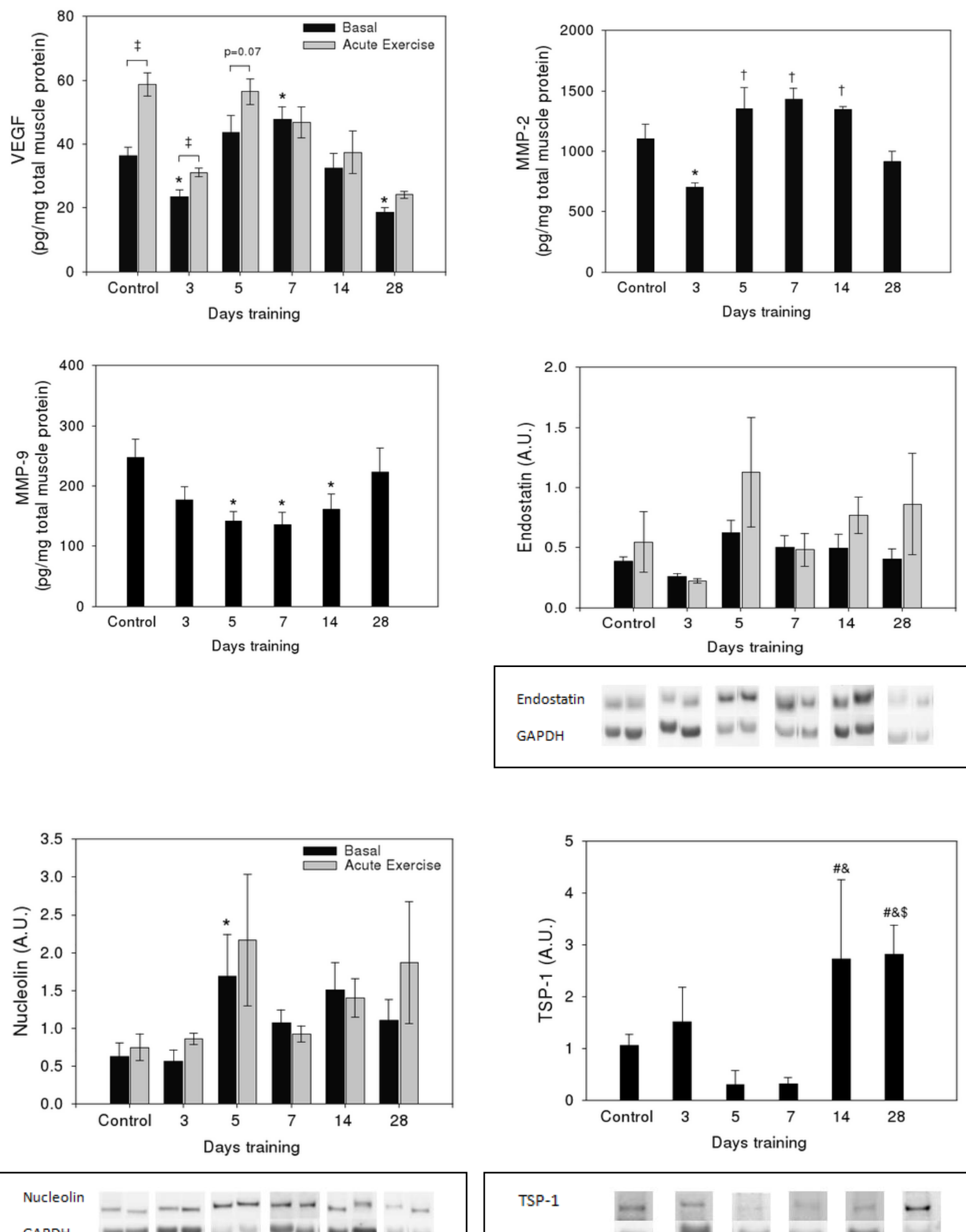

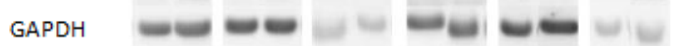

TSP-1

GAPDH

Figure 3. 


\section{CHAPTER 3:}

Effects of Detraining on the Temporal Expression of Key Positive and Negative Angioregulatory Proteins in Skeletal Muscle

Sara A. Olenich ${ }^{1}$, Gerald N. Audet ${ }^{1}$, Kathleen A. Roberts ${ }^{1,2}$, and I. Mark Olfert ${ }^{1}$

(1)West Virginia University School of Medicine Center for Cardiovascular and Respiratory Sciences

Division of Exercise Physiology

One Medical Center Dr.

Morgantown, WV 26506

(2)West Virginia Wesleyan College

59 College Avenue

Buckhannon, WV 26201

Short Title: Molecular responses to detraining

Total words:

Corresponding Author:

Mark Olfert, Ph.D.

West Virginia University School of Medicine

Center for Cardiovascular and Respiratory Sciences

Division of Exercise Physiology

One Medical Center Dr.

Morgantown, WV 26506-9105

Tel 304-293-7597

Fax 304-293-5513

molfert@hsc.wvu.edu

ABSTRACT (294 WORDS) 
Temporal expression of positive and negative angiogenic factors in response to detraining is poorly understood. We report the protein expression of anti-angiogenic peptides (thrombospondin-1, TSP-1; and endostatin) as well as pro-angiogenic mitogens (vascular endothelial growth factor, VEGF; matrix metalloproteinases-2 and MMP-9), and nucleolin (a nuclear protein involved with synthesis and maturation of ribosomes) in response to detraining in triceps surae muscles of mice. Mice were allowed to voluntarily train for 21 days, and then basal and acute response to exercise were evaluated at 1-, 7-, 14- and 28-days detraining (D1, D7, D14, D28 groups, respectively, $n=12$ /group). Training resulted in the expected increases muscle capillary-to-fiber ratio (C:F), maximal run test and basal expression of VEGF and MMP-9 $(p<0.05)$, as seen in the D1 group (i.e. trained control) mice. After 7 days of detraining (D7), C:F returned to control levels, but both basal VEGF and TSP-1 were elevated $(p<0.05)$. In D14 and D28 group mice, TSP-1 protein fell to baseline levels, but VEGF remained elevated in gastrocnemius, but not the soleus or plantaris, of D14 mice. Endostatin tended to decrease with detraining, resulting in significantly lower basal levels by D14 and D28 for most muscles compared to controls. Nucleolin protein expression was inconsistent across muscle, with increases at D1 in the plantaris, D7 in the soleus and D14 in the gastrocnemius. The response of VEGF to acute exercise was blunted with training, and surprisingly was still blunted even after 28 days of detraining, despite the return of muscle capillarization to baseline levels. These data suggest that TSP-1 may be an important mediator in capillary regression with detraining. The responses of VEGF and MMPs to detraining did not change in correlation to changes in muscle capillarity, suggesting that pro-angiogenic regulators may not play a prominent role in regulating physiological decreases in muscle capillarity. 


\section{INTRODUCTION}

Skeletal muscle has a tremendous plasticity to adapt in response to exercise. One important adaptation is increasing capillarity in the working muscle to help reduce the metabolic stress imposed during physical activity (Andersen \& Henriksson, 1977). While the molecular underpinnings of capillary expansion (i.e. angiogenesis) have been intensely studied, our understanding of the mechanisms involving physiologic mediated regression of muscle capillaries (i.e. physical deconditioning after cessation of training) is less well understood.

It is generally accepted that exercise induced skeletal muscle angiogenesis is controlled by a balance between positive and negative factors (Egginton, 2009; Olfert \& Birot, 2011). Among the angioregulatory factors believed to be important in skeletal muscle are: proangiogenic vascular endothelial growth factor (VEGF), and anti-angiogenic thrombospondin-1 (TSP-1), both of which have been shown to exhibit robust mRNA response to an acute bout of exercise in rodents and humans (Breen et al., 1996; Gustafsson et al., 1999; Richardson et al., 1999; Gavin \& Wagner, 2001; Olfert et al., 2006; Hoier et al., 2012). Gene deletion strategies in mice have shown that muscle-specific loss of VEGF decreases muscle capillarity (Tang et al., 2004; Olfert et al., 2009), whereas a loss of TSP-1 increases basal muscle capillarity (Malek \& Olfert, 2009). Moreover, lack of skeletal muscle VEGF has been reported to prevent training induced skeletal muscle angiogenesis (Olfert et al., 2010), demonstrating the myocyte VEGF is essential to capillary expansion in response to physical exercise. But to what extent VEGF, TSP-1, and/or other key angiogenic regulators influence capillary regression upon cessation of training (i.e. detraining) is less well understood.

Unlike tumor angiogenesis, which represents an uncontrolled and dysregulated expansion of blood vessels (Folkman, 1995; Hanahan \& Folkman, 1996), physiologicallymediated angiogenesis (such as that seen in the female reproductive cycle or response to exercise training) is a tightly-controlled, highly-coordinated multiple-step process regulating endothelial cell proliferation, extracellular matrix remodeling and vessel wall 
assembly/stabilization (Iruela-Arispe et al., 1995; Prior et al., 2004). It is conceivable that timing of pro- and anti-angiogenic regulator expression plays an important part in the successful execution of this highly choreographed process (Egginton, 2009; Olfert \& Birot, 2011). Likewise, it could also be expected that the temporal expression of angiogenic regulating peptides may also determine the physiologic regression of skeletal muscle capillary in response to detraining.

At present, we are aware of only 2 studies that report the expression of VEGF following detraining in skeletal muscle. Malek et al. report that VEGF protein in the plantaris muscle has not returned to control levels after 7 days of detraining in previously trained rats (Malek et al., 2010). And, Huttemann et al. (2012) report (in mice which trained for 5 weeks) that VEGF protein in the gastrocnemius muscle remained elevated after 14-days of detraining. They also report that TSP-1 was significantly decreased with training but returned to untrained control levels following 14-days of detraining (Huttemann et al., 2012), suggesting that altered expression of TSP-1 (and not VEGF per se) may be responsible for the detraining mediated losses in skeletal muscle capillarity. Taken together, these 2 studies suggest the temporal response of VEGF and TSP-1 may differ with time spent detraining. But given that only one time point was assessed in either study, it is difficult to characterize and interpret any temporal effects. Moreover, neither study included assessment of other angiogenic regulators, such as matrix metalloproteinases (MMPs) or endostatin, which are also important in regulating angiogenesis. For example, MMP-2 and MMP-9 may be especially important because they are necessary to destabilize the basement membrane of the existing vasculature and the extracellular matrix (ECM) (Nagase \& Woessner, 1999; Haas et al., 2000). Endostatin, which is a 22-kD anti-angiogenic fragment of collagen XVIII cleaved by MMPs, is capable of inhibiting VEGF and up regulating TSP-1 (Yamaguchi et al., 1999; Kim et al., 2000; Kim et al., 2002; Volpert et al., 2002; Hu et al., 2012) and therefore could have a significant influence on capillary regression. At present, however, nothing is known about the expression of endostatin in the context of detraining. Also of interest, is the finding that endostatin activity may be dependent on 
nucleolin -- a nuclear protein found on the surface of endothelial cells undergoing proliferation and which is also important for processing ribosome transcription, editing and assembly, and in the import/export of proteins to the nuclear membrane (Lapeyre et al., 1987; Srivastava \& Pollard, 1999). Nucleolin and endostatin appear to co-localize on the cell surface, which can lead to endostatin transport to the nuclei (via nucleolin) where its inhibiting effects on cell proliferation can take place (Shi et al., 2007). Thus the temporal expression of nucleolin and endostatin could be an important pathway regulating the physiologic adaptation of microvessels within skeletal muscle.

We hypothesize that detraining will preferentially increase the temporal expression of key negative angiogenic regulators (such as TSP-1, endostatin) with little influence on basal expression of pro-angiogenic regulators (such as VEGF, MMP-2, MMP-9, nucleolin), leading to regression of muscle capillaries and reduced exercise performance with detraining. To test this hypothesis, we provided mice with 24-hour access to running wheels for 21 days (a voluntary training paradigm known to significantly increase hindlimb skeletal muscle capillarity). Then muscle capillarity, as well as the basal and acute protein response to exercise, were separately examined after 1-, 7-, 14- and 28-days of detraining (D1, D7, D14 and D28, respectively).

\section{MATERIALS AND METHODS}

Mice: Male C57BL/6 mice were purchased from Jackson Laboratories (Stock\# 000664, Bar Harbor, ME). Mice were housed in 12hr:12hr light:dark cycle with provided free access to food and tap water. Experiments and animal care/handling were performed in accordance to PHS Animal Welfare Act and received approval by West Virginia University Institutional Animal Care and Use Committee.

Training and Deconditioning: Sixty C57BL/6 mice were randomly assigned into a control group (no training) or one of four groups corresponding to 1-, 7-, 14- and 28-days detraining ( $n=12 /$ group) after 21 days of training. For training, all mice (except control mice) were given 24- 
hour access to running wheels to voluntarily train for 21 days prior to detraining. Mice were individually housed in cages containing a running wheel (4.5" Mouse Wheel, Respironics, Bend, OR) connected to a bike computer (Cateye Strada CC-RD100N, CatEye North America, Boulder, CO) which digitally measured and recorded distance, running time, mean running speed, and maximum running speed. Performance over a 24-hour period was recorded each day for each animal. Upon completion of training the wheels were removed from the cages and mice were detrained according to the respective temporal assignment (i.e. D1, D7, D14 and D28). Basal muscle expression for $D 1(n=6)$ was evaluated after one full-day without access to the running wheel, and therefore essentially serves as a trained control group. The day without access to the running wheel was to minimize any acute response to wheel running exercise. The remaining detraining groups were sacrificed on the day indicated for each respective group (i.e. D7, D14, D28; n=6/group).

Acute Exercise: To examine the angiogenic responses to acute exercise at each of the time points (i.e. Control, D1, D7, D14, D28), half of the mice ( $n=6 /$ group) performed a standardized single 1 -hour acute bout of exercise at $20 \mathrm{~m} / \mathrm{min}$ with $10^{\circ}$ incline on a rodent treadmill (Columbus Instruments, Exer-6M Treadmill, Columbus, $\mathrm{OH}$ ), and were then sacrificed 4 hours after the acute exercise bout. Like the basal muscle expression group, acute exercise was performed following a full day without access to the running wheel to minimize any response from wheel running exercise. For all mice, muscle from the left triceps surae (gastrocnemius (GA), plantaris (PLT), soleus (SOL)) were extracted for molecular analysis and muscle from the right triceps surae for morphometric analysis.

Maximal Run Test: A maximal running speed test was used to measure aerobic performance prior to training, on the final day of training, and one day prior to completion of detraining for each respective group. All mice experienced a 24-30 hour rest period (with no wheel access) prior to sacrifice examining basal or acute exercise responses. Maximal running was performed on a mouse treadmill (Columbus Instruments, Exer-6M Treadmill, Columbus, 
$\mathrm{OH}$ ) at a $10^{\circ}$ incline. Warm up was 5 minutes at $4 \mathrm{~m} / \mathrm{min}$, and then increased by $2 \mathrm{~m} / \mathrm{min}$ every 30 seconds until test termination defined by the inability of the animal to continue running on the belt despite encouragement.

Tissue collection and processing. All mice were anesthetized with xylazine/ketamine (Ketaject $100 \mathrm{mg} / \mathrm{kg}$, Xylazine $5 \mathrm{mg} / \mathrm{kg}$ ) via intraperitoneal injection. Once anesthetized the gastrocnemius, plantaris, and soleus muscles from the left leg were carefully removed at its respective origin and insertion attachment, weighed, and immediately flash frozen in liquid nitrogen for protein analyses. Gastrocnemius, plantaris, and soleus from the right leg were then removed in the same manner as the left leg, the mid-belly of the muscles were carefully resected and positioned transversely on a cork base and carefully embedded in tissue freezing medium (TBS Tissue Freezing Medium ${ }^{\mathrm{TM}}$, Triangle Biomedical Sciences, Durham, NC, USA) and immediately flash-frozen in liquid nitrogen cooled isopentane (2-methylbutane, Fisher Chemical O3551-4, Fisher Scientific). All muscle samples were stored at $-80 \mathrm{C}$ until processed for molecular or morphometric analyses.

Homogenization: Skeletal muscle was homogenized using a lysis buffer containing 50 $\mathrm{mM}$ Tris/ $\mathrm{HCl}(\mathrm{pH} 7.4), 150 \mathrm{mM} \mathrm{NaCl}, 0.5 \%$ Triton X-100, and protease inhibitors (Complete ${ }^{\mathrm{TM}}$ Tablet, Roche Applied Science, Indianapolis, IN). Homogenates were centrifuged at $4^{\circ} \mathrm{C}$, $14000 \mathrm{~g}$ for 10 minutes and supernatants were then be removed and placed in new tubes. Total protein was measured by the Bradford Assay (Coomassie Plus \#23236 Assay Pierce Biotechnology, Rockford, IL).

Protein Analysis: Mouse VEGF, MMP-2, and MMP-9 protein levels were measured from $100 \mu \mathrm{g}$ of total protein using a commercially available ELISA kits (VEGF \#MMV00, MMP-9 \#MMPT90 from R\&D Systems, Minneapolis, MN and MMP-2 \#ELM-MMP2-001 from RayBiotech, Norcross, GA). All analyses were performed according to the manufacturer's specification and optical densities were measured using a microplate reader (Bio Rad Model 550, Global Medical Instrumentation Inc., Ramsey, Minnesota). For western analysis, samples 


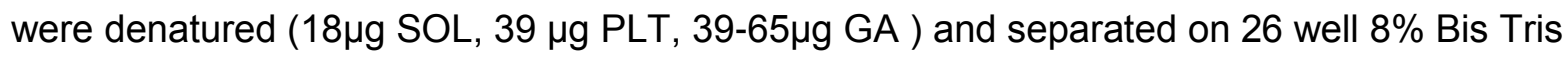
Midi Gel (Novex WG1003BX10, Carlsbad, CA) and blotted onto a 0.45 $\mu \mathrm{m}$ nitrocellulose membrane (nitrocellulose membrane \#88018 Thermo Scientific). Membrane was blocked with $5 \%$ fat-free milk in TBS and then membrane was cut twice to yield three membrane strips (TSP1/nucleolin, GAPDH, endostatin) and probed with respective antibodies TSP-1

(Thrombospondin 1 (A6.1): sc-59887, Santa Cruz Biotechnology, Santa Cruz, CA); nucleolin (nucleolin antibody \#A300-711A, Bethyl Laboratories, Montgomery, TX); GAPDH (mouse antiGAPDH \#39-8600, Invitrogen, Camarillo, CA); endostatin (anti-mouse endostatin antibody \#AF570 R\&D Systems, Minneapolis, MN). Nucleolin was probed after TSP-1 had been measured. Equal loading was verified and normalized by the loading control, GAPDH. Chemiluminescence detection allowed for visualization of proteins (Pierce ECL Western Blotting Substrate, \#32209, Rockford, IL), images of blots were taken using Genesnap software (version 7.01; Syngene). Protein expression was quantified by using NIH ImageJ Software (version 1.62) and expressed as densitometric arbitrary units (a.u.). Control samples were loaded on each gel to normalize groups.

Histochemistry. Corks containing frozen muscles embedded in tissue freezing medium were cut into serial 8-10 $\mu \mathrm{m}$ transverse sections using a microcyrotome at $-20^{\circ} \mathrm{C}$ (Leica CM1850, Leica Biosystems, Buffalo Grove, IL) and mounted on glass slides (Colormark* Plus Adhesion Slides, \#CM-7951, Thermo Scientific) for histochemical analysis of muscle morphometry. Great care was taken to ensure that the widest part of the muscle was sectioned and that cuts were made perpendicular to the orientation of the fibers. Muscle capillarity was determined by measuring capillary-to-fiber ratio (C:F) by using the alkaline phosphatase and dipeptidylpeptidase capillary staining method (Mrazkova et al., 1986). Stained muscle sections were viewed by light microscopy and digitally imaged at 20x magnification. Muscle capillarity was assessed from deep and superficial regions within the gastrocnemius. Deep regions are defined as the inner half of the gastrocnemius muscle (closest in proximity to the plantaris and 
soleus muscles), while superficial regions are comprised of the outer half of the gastrocnemius (closest in proximity to the skin). Up to 15 digital images were obtained from each quadrant in a pseudo-random selection process. Images were selected if they did not overlap with any previous image selected and did not violate the boundary used to demarcate the quadrant being imaged. The imaging technician was instructed not to "frame" each image per se, but rather accept the random image position (after moving the microscope stage) within each quadrant, provided they do not violate the image selection criteria above.

Statistical Analyses: All data shown are mean \pm SEM, unless otherwise indicated. Changes in protein expression, exercise performance during training (i.e. daily distance run, time running, average speed and maximal speed), and muscle capillarity (i.e. C:F) were analyzed using one-way ANOVA. A repeated measure ANOVA was used for multiple measures of maximal exercise performance testing. The combined effects of training (i.e. groups trained for different days) and the angiogenic factor response to acute exercise (i.e. basal vs. acute exercise) for proteins were separately determined using a $2 \times 2$ factorial ANOVA. When a main effect was observed, post hoc testing with a Fisher's PLSD was used to examine group differences. All statistics were performed using Statview Statistical Software package (v5.0.01, SAS Institute Inc., Cary, NC). Significance was set at $\alpha$ level of $p=0.05$.

\section{$\underline{\text { RESULTS }}$}

Animal body mass and muscle characteristics. There was no significant difference in age, body mass or hindlind muscle mass (when normalized to body mass) compared to the control group (Table 1). When compared to control mice, training increased capillary-to-fiber ratio $(C: F)$ in the gastrocnemius $(p=0.04)$, plantaris $(p=0.04)$, and soleus $(p=0.04)$ (Table 1$)$. In all muscles, C:F returned to control levels after 7 days of detraining and stayed there with 14 and 28 days of detraining. 
Training behavior and aerobic performance. When comparing voluntary wheel running behavior across all groups there was no significant difference in time spent running (ranging from an average of 306 to 392 minutes each day, Table 1). However D14 and D28 mice exhibited higher average running speed, average distance run per day, and higher maximal wheel training speed compared to D7 mice $(p<0.05)$, but not compared to D1 mice (Table 1$)$.

Aerobic performance measured by a maximal running test before training, at the end of training and at the end of detraining, showed that all groups universally increased maximal running speed with training, which returned to control (untrained) levels in all detraining groups (Figure 1).

\section{Basal Protein Expresison.}

Gastrocnemius. Shown in Figure 2a, training (i.e. D1 time point) resulted in an increase of basal VEGF $(p=0.02)$ and MMP-9 $(p=0.04)$, all other factors remained unchanged when compared to the control group. After 7 days of detraining (D7), VEGF $(p<0.01)$ remained elevated and TSP-1 $(p<0.01)$ increased. Following 14 days of detraining (D14), basal VEGF $(p<0.01)$ levels were still elevated while TSP-1 fell to baseline. Nucleolin was also significantly elevated at D14 compared to controls. At 28 days post training (D28), basal VEGF returned to baseline, TSP-1 remained at baseline levels and endostatin $(p<0.01)$, MMP-2 $(p<0.01)$ and MMP-9 $(p=0.03)$ had all decreased compared to controls.

Plantaris. Shown in Figure 2b, training (D1) resulted in an increase of basal VEGF $(p<0.01)$, nucleolin $(p=0.03)$, and TSP-1 $(p<0.01)$. Endostatin did not change when compared to the control group. After 7 days of training (D7), VEGF $(p<0.01)$ and TSP-1 $(p<0.01)$ remained elevated. Nucleolin decreased and endostatin remained unchanged. Following 14 days of detraining (D14), VEGF fell to control levels while endostatin $(p<0.01)$ was significantly diminished, TSP-1 and nucleolin remained unchanged. At 28 days post training (D28), endostatin $(p<0.01)$ remained low, VEGF, TSP-1, and nucleolin were not different from controls. 
Soleus. Shown in Figure 2c, training (D1) resulted in a decrease only of endostatin $(p=0.02)$ when compared to the control group. After 7 days of detraining (D7), endostatin returned to control levels while VEGF $(p<0.01)$, nucleolin $(p<0.01)$, and TSP-1 $(p<0.04)$ were all significantly increased. Following 14 days of detraining, endostatin $(p<0.01)$ was increased while VEGF, nucleolin and TSP-1 returned to control levels. At 28 days post training VEGF was elevated $(p<0.01)$, endostatin $(p<0.01)$ was decreased and TSP-1 and nucleolin were not different from control levels.

\section{Protein response to Acute Exercise.}

VEGF protein responded to acute exercise in the control group in the plantaris $(p<0.04)$ and soleus $(p<0.01)$, and to a lesser extent in gastrocnemius (Figure 3$)$. This response was blunted with training (D1) and remained blunted after 7, 14 or 28 days of detraining in the PLT or SOL. Endostatin had a group $x$ acute exercise interaction in the SOL with $(p<0.05)$ and to lesser extent in the PLT ( $p=0.057)$, but did not have an increase of protein following acute exercise in any of the muscles. Nucleolin tended to increase in response to acute exercise in all three muscles of control mice, this response was blunted in trained mice, and remained blunted with detraining. Furthermore, a significant group $x$ acute exercise interaction $(p<0.05)$ was only observed in GA and PLT for nucleolin (Figure 3). Responses for TSP-1, MMP-2 and MMP-9 are not reported here because these factors have been shown to respond to acute exercise at much later time point (than 4-hours post exercise) used in this study.

\section{DISCUSSION}

The principal finding of this study is that detraining losses in skeletal muscle capillarity, occurring after 7 days detraining, are associated with elevated basal expression of TSP-1. Surprisingly, basal VEGF protein levels were also elevated after 7-days detraining. Endostatin was not elevated at 7-days post training compared to controls, but rather demonstrated a 
tendency to decrease with greater time spent detraining. These data suggest that losses in skeletal muscle capillarity (due to physical deconditioning) may be more dependent on the expression of TSP-1 and/or other negative regulators, rather than reductions in positive angiogenic regulators, such as VEGF.

\section{Training response and muscle capillarization}

Our data show some variability in the voluntary wheel running behavior between mice in our respective groups, where D14 and D28 mice exhibit greater daily averages in distance and speed compared to D7. But compared to trained (D1) mice, D14 and D28 were not different. It should also be noted that average time spent running was similar across all groups. Importantly, the training performed (as assessed in the D1 group) significantly increased muscle capillarity in the hindlimb muscles (Table 1) and resulted in similar increases in aerobic performance capability for all groups (Figure 1). These increases universally returned back to baseline levels in each detrained group (i.e. D7, D14, D28). Thus, although it was not possible to measure muscle capillarity after training in the D7, D14, or D28 groups, based on the overall running behavior and performance it is reasonable to expect similar capillary adaptation to training as that seen and reported for D1 (training control) mice.

Consistent with training induced capillary adaptation, as well as previous reports from training studies (Carmeli et al., 2007; Rullman et al., 2009; Huttemann et al., 2012), we observed an elevated basal expression of VEGF and MMP-9 in trained (D1) mice, with one exception. VEGF was not elevated in the soleus muscle in D1 mice. TSP-1 was unchanged in trained (D1) mice, except in plantaris muscle. The lack of elevated basal VEGF in the soleus muscle is not surprising and is in line with a study by Birot et al which demonstrated that VEGF mRNA was up regulated to a greater extent in type Ilb myofibers than type I or Ila, most likely due to their glycolytic nature (Birot et al., 2003). The soleus muscle is highly oxidative with a rich vascular supply and therefore may not have the same oxygen demand/deficit created by 
exercise to trigger a significant increase in VEGF as that seen in the plantaris and gastrocnemius.

However, the reason for elevated basal TSP-1 expression in the D1 plantaris, but not the gastrocnemius and soleus is less clear. Previous reports have shown TSP-1 mRNA is increased in response to acute exercise, but this response is eventually lost with repeated or chronic exercise (Olfert et al., 2006). Data from our previous training study (unpublished data, see Training Study) show no significant difference in basal TSP-1 protein expression in the gastrocnemius muscle after 14 to 28 days of voluntary wheel training compared to untrained controls, which supports the current observation in the gastrocnemius and soleus muscle of the animals in this study. Thus, whether the response in the plantaris is a residual effect of training or specific to the plantaris is presently unclear. However, it is possible, as previously noted for the VEGF response to training, that this finding could be due the greater glycolytic fiber type composition of the plantaris muscle compared to the overall greater oxidative nature seen in the soleus and the mixed gastrocnemius muscles. This is supported by evidence of the lower VEGF/TSP-1 ratio (suggesting higher TSP-1 relative to VEGF) reported in plantaris muscle compared to the soleus muscle during hindlimb unloading (Roudier et al., 2010). Greater basal TSP-1 protein levels have also been reported in the plantaris compared to gastrocnemius (Audet et al., 2011).

Endostatin was largely unaltered in response to training, except for a decreased expression level in the D1 soleus muscle $(p<0.05)$. The lack of response from endostatin is consistent with the response in gastrocnemius muscle from our previous Training Study (unpublished data, see Training Study). Because the actions of endostatin may be regulated in part by nucleolin, we also assessed nucleolin levels in response to detraining. We show that nucleolin tends to be up regulated in response to acute exercise, which is in accordance with the acute response to exercise we report in our Training Study (unpublished data, see Training Study). Training induced (D1) increase in nucleolin was only seen in the plantaris muscle. An 
argument could be made to that, this too, may involve the greater glycolytic nature of the plantaris and mixed gastrocnemius compared to the more oxidative fiber type composition of the soleus muscle. But further studies are needed to examine nucleolin's role in skeletal muscle angiogenesis before the importance and/or contribution of nucleolin will be clearly understood.

\section{Detraining and protein expression in skeletal muscles}

In this study we show similar trends across the muscles examined (GA, PLT, SOL) but each muscle also appears to have its own unique temporal response to exercise. In all muscles examined, there was a statistically significant increase in basal expression of VEGF and TSP-1, concurrent with a decrease in muscle capillarity at after 7 day detraining (D7) compared to trained control (D1) mice. We would not have predicted that skeletal muscle capillarity would have returned to baseline conditions with elevated VEGF levels given it prominent role in stimulating angiogenesis. It curious to note that basal VEGF in the gastrocnemius muscle remained elevated (while TSP-1 returned to baseline) after 14 days of detraining, perhaps suggesting a window of heighten sensitivity in response to capillary regression and/or dependence on a cofactor to trigger new blood vessel growth. Only after 28 days of detraining did VEGF return to basal levels. At this same 28 day time point, endostatin, MMP-2, and MMP-9 were all reduced. We speculate endostatin is likely reduced due to lower MMPs and may serve to remove its inhibitory influence in response to acute exercise, and increase the sensitivity of VEGF to an exercise stimulus.

In the plantaris, both endostatin and nucleolin tended to decrease with increasing number of days detraining with significantly lower expression at D28 compared to controls (Figure 2b). Unlike the gastrocnemius muscle, after 14 days of detraining, VEGF returned to control levels and while endostatin tended to diminished through 28 days of detraining. Although VEGF levels are low (not in favor of angiogenesis), endostatin levels are also low (favoring angiogenesis) perhaps keeping a balance between positive and negative angiogenic factors. 
Overall, the plantaris appears to have an accelerated molecular response when compared to the gastrocnemius (i.e. VEGF returns to basal levels and endostatin levels are reduced faster which may be due to differences in fiber type). Unfortunately, due to small muscle size and insufficient sample for analysis, we were not able to measure MMP-2 and MMP-9 in either the soleus or plantaris.

In the soleus, the only protein to be altered by training was endostatin which was reduced from D1. Likewise, endostatin levels have been reported to be chronically low in other oxidative muscles such as the heart (Gu et al., 2006). It was only after D7 that endostatin returned to control levels. In stark contrast, VEGF, TSP-1 and nucleolin (as seen in the PLT) were all significantly up-regulated, suggest that the D7 time point could be a tipping point in the soleus with all these proteins at play. After D14, endostatin increased while all other proteins that were elevated returned to control levels. At D28, endostatin subsided below control levels and VEGF was again elevated.

Taken together, the main theme that emerges is that TSP-1 may play a prominent role in capillary regression associated with detraining. This is consistent with evidence that TSP-1 is involved in capillary regression seen during menstrual cycle (Iruela-Arispe et al., 1996; Slater \& Murphy, 1999). Here, VEGF is has been shown to be localized in capillaries and was most strongly detected in the late proliferative and secretory phase (Bausero et al., 1998) while TSP1 has been associated with low capillary growth in the secretory phase (Iruela-Arispe et al., 1996). So, it may not be unexpected that despite having elevated VEGF levels at D7 in each hindlimb muscle examined, muscle capillary was reduced at D7. Unfortunately, the temporal resolution of our detraining time course does not allow us to pin point when TSP-1 was first upregulated between $D 1$ and D7 or when the reversal of the angiogenic gains made by training occurred, but based on these data it is clear that morphological changes in capillarity are fully affected by 7 days in this training/detraining paradigm. 


\section{Response to Acute Exercise Following Detraining}

In the current study, we also evaluated the acute exercise response of angiogenic regulators as a means to evaluate the effect of detraining and changes in muscle capillarityspecifically, when/what are the events that lead to a detrained muscle to return to pre-trained conditions. Based on prior evidence (unpublished data, see Training Study), we know that peak response for VEGF, endostatin and nucleolin protein expression occurs $\sim 4$ hours post exercise, therefore this time point was selected to harvest muscle to access angiogenic protein response to acute exercise with the temporal response to detraining. Since the peak protein responses for TSP-1, MMP-2, and MMP-9 s did not occur at this time point, there is limited, if any, interpretative value to be gained from assessing the acute response of these factors in the current study.

As observed in our Training Study (unpublished data, see Training Study), as well as in other publications (Olfert et al., 2001; Hoier et al., 2012), we find acute exercise increases skeletal muscle VEGF protein levels in plantaris and soleus muscles. Surprisingly, this response did not prove statistically significant in the gastrocnemius despite a consistent trend for VEGF to increase. Nonetheless, the VEGF response to exercise is well documented in literature and is established as a prime process orchestrating skeletal muscle angiogenesis. Since it has been reported that responsiveness of VEGF mRNA (Olfert et al., 2001) and protein to acute exercise is lost or blunted with training (Malek et al., 2010), a key question this study aimed to answer is when would this acute response to exercise return with detraining. Consistent with previous reports, the D1 mice exhibited a blunted (or absent) VEGF protein response to acute exercise. Unexpectedly, the acute VEGF response to exercise never significantly reemerged even after 28 days of detraining in any of the muscles, except for one case (i.e. D14 in the GA). This is surprising because an underlying theory for the blunted response of VEGF with training was thought to be due to greater number of capillaries (induced by training) satisfying the metabolic stress associated with chronic exercise. The present finding 
argues against this idea suggesting that some other mechanism is likely attenuating the VEGF response to acute exercise and keeping it blunt despite the losses of capillaries occurring with detraining.

In contrast to our data, Malek et al. report the acute VEGF response to exercise had returned after 7 days of detraining in the plantaris and the soleus muscles of rats who treadmill trained 3 days a week for 10 weeks (Malek et al., 2010). In addition to using a different animal model from the current study, the training regimen was longer in duration, and used lower frequency but higher exercise intensity $(50-80 \%$ of maximal work rate) than that performed by our mice which voluntarily performed high daily frequency but low intensity exercise (at $~ 34-$ $40 \%$ of their maximal exercise capacity). Thus it could be that high-volume-low-intensity exercise has a different effect on angiogenic regulators that low-volume-higher-intensity exercise. This notion is supported by recent evidence in humans, where intense intermittent, low volume, exercise has been reported to be a weaker stimulus for angiogenesis than constant moderate intensity exercise (Hoier et al., 2013).

We have previously found that endostatin and nucleolin protein levels were up-regulated at 4 hours post-acute exercise, but this was not the case for endostatin in our untrained control mice in the present study. In both the gastrocnemius and plantaris, nucleolin demonstrated significant group $x$ acute exercise interaction effect $(p<0.05)$ with a tendency of elevated response in untrained control mice in all three muscles (Figure 3). Despite this post-hoc analysis for acute response did not prove statistically significant. An explanation for this discrepancy between these data and our previous data (unpublished data, see Training Study) remains unclear, but could raise questions whether the response of either endostatin or nucleolin represents a significant biological response to exercise. It is important to note, at least in the case of endostatin, that it is not a transcriptionally-regulated peptide but rather a fragment from a larger extracellular matrix molecule (i.e. collagen XVIII). Thus the inconsistent endostatin response we observed could likely be associated with the responses of other factors, such as 
MMP-2 or MMP-9 which influence proteolytic activity within the extracellular matrix. Unfortunately, due to the small mass and total protein available we did not have sufficient sample to make an assessment of MMP-2 or MMP-9 in either the plantaris or soleus muscles.

In conclusion, this study has demonstrated the temporal response of key positive and negative angiogenic regulators in the gastrocnemius, plantaris, and soleus muscles in response to detraining. We have shown that the VEGF protein response to acute exercise remains blunted after 28 days of training and that elevated TSP-1 and VEGF expression are associated with capillary regression occurring after 7 days of detraining. These data suggest the physiologic regression of muscle capillaries may be influence greater by negative angiogenic regulators (such as TSP-1) rather than positive angiogenic regulators (such as VEGF). Future studies will be needed to determine when the acute VEGF response to exercise returns, while shorter term detraining studies are needed to identify how soon with detraining TSP-1 protein expression starts to increase. 


\section{ACKNOWLEDGEMENTS}

Funding support was provided, in part by, West Virginia University Research Funding and Development Grant (Olfert), American Heart Association 10BGIA3630002 (Olfert), WVUINBRE grant supported by NIH 5P20RR016477 (Olfert \& Roberts); and West Virginia University Cardiovascular and Respiratory Training Grant NIH 5T32HL090610 (Audet).

Authors have no conflict of interest to disclose. 


\section{REFERENCES}

Andersen P \& Henriksson J (1977). Capillary supply of the quadriceps femoris muscle of man: adaptive response to exercise. J Physiol 270, 677-690.

Audet GN, Meek TH, Garland Jr T \& Olfert IM (2011). Expression of angiogenic regulators and skeletal muscle capillarity in selectively bred high aerobic capacity mice. Experimental Physiology 96, 1138-1150.

Bausero P, Cavaille F, Meduri G, Freitas S \& Perrot-Applanat M (1998). Paracrine action of vascular endothelial growth factor in the human endometrium: production and target sites, and hormonal regulation. Angiogenesis 2, 167-182.

Birot OJ, Koulmann N, Peinnequin A \& Bigard XA (2003). Exercise-induced expression of vascular endothelial growth factor mRNA in rat skeletal muscle is dependent on fibre type. J Physiol 552, 213-221.

Breen EC, Johnson EC, Wagner H, Tseng HM, Sung LA \& Wagner PD (1996). Angiogenic growth factor mRNA responses in muscle to a single bout of exercise. J Appl Physio/ 81, 355-361.

Carmeli E, Haimovitz T \& Nemcovsky EC (2007). Cathepsin D and MMP-9 activity increase following a high intensity exercise in hind limb muscles of young rats. J Basic Clin Physiol Pharmacol 18, 79-86.

Egginton S (2009). Invited review: activity-induced angiogenesis. Pflugers Arch 457, 963-977.

Folkman J (1995). Angiogenesis in cancer, vascular, rheumatoid and other diseases. Nature Med. 1, 27-31.

Gavin TP \& Wagner PD (2001). Effect of short-term exercise training on angiogenic growth factor gene responses in rat. J. Appl. Physiol. 90, 1219-1226.

Gu J-W, Shparago M, Tan W \& Bailey AP (2006). Tissue Endostatin Correlates Inversely with Capillary Network in Rat Heart and Skeletal Muscles. Angiogenesis 9, 93-99.

Gustafsson T, Puntschart A, Kaijser L, Jansson E \& Sundberg CJ (1999). Exercise-induced expression of angiogenesis-related transcription and growth factors in human skeletal muscle. Am J Physiol 276, H679-685. 
Haas TL, Milkiewicz M, Davis SJ, Zhou AL, Egginton S, Brown MD, Madri JA \& Hudlicka O (2000). Matrix metalloproteinase activity is required for activity-induced angiogenesis in rat skeletal muscle. Am J Physiol Heart Circ Physiol 279, H1540-1547.

Hanahan D \& Folkman J (1996). Patterns and Emerging Mechanisms of the Angiogenic Switch during Tumorigenesis. Cell 86, 353-364.

Hoier B, Nordsborg N, Andersen S, Jensen L, Nybo L, Bangsbo J \& Hellsten Y (2012). Pro- and anti-angiogenic factors in human skeletal muscle in response to acute exercise and training. J Physiol 590, 595-606.

Hoier B, Passos M, Bangsbo J \& Hellsten Y (2013). Intense intermittent exercise provides weak stimulus for vascular endothelial growth factor secretion and capillary growth in skeletal muscle. Exp Physiol 98, 585-597.

Hu W, Xia LJ, Chen FH, Wu FR, Tang J, Chen CZ, Jiang S \& Chen HH (2012). Recombinant human endostatin inhibits adjuvant arthritis by down-regulating VEGF expression and suppression of TNF-alpha, IL-1beta production. Inflamm Res 61, 827-835.

Huttemann M, Lee I \& Malek MH (2012). (-)-Epicatechin maintains endurance training adaptation in mice after 14 days of detraining. Faseb $J$ 26, 1413-1422.

Iruela-Arispe ML, Porter P, Bornstein P \& Sage EH (1995). Thrombospondin-1, an inhibitor of angiogenesis, is regulated by progesterone in the human endometrium. J. Clin. Invest. 97, 403-412.

Iruela-Arispe ML, Porter P, Bornstein P \& Sage EH (1996). Thrombospondin-1, an inhibitor of angiogenesis, is regulated by progesterone in the human endometrium. J Clin Invest 97, 403-412.

Kim YM, Hwang S, Pyun BJ, Kim TY, Lee ST, Gho YS \& Kwon YG (2002). Endostatin blocks vascular endothelial growth factor-mediated signaling via direct interaction with $\mathrm{KDR} / \mathrm{Flk}$ 1. J Biol Chem 277, 27872-27879.

Kim YM, Jang JW, Lee OH, Yeon J, Choi EY, Kim KW, Lee ST \& Kwon YG (2000). Endostatin inhibits endothelial and tumor cellular invasion by blocking the activation and catalytic activity of matrix metalloproteinase. Cancer Res 60, 5410-5413.

Lapeyre B, Bourbon H \& Amalric F (1987). Nucleolin, the major nucleolar protein of growing eukaryotic cells: an unusual protein structure revealed by the nucleotide sequence. Proc Natl Acad Sci U S A 84, 1472-1476. 
Malek MH \& Olfert IM (2009). Global deletion of thrombospondin-1 increases cardiac and skeletal muscle capillarity and exercise capacity. Exp Physiol 94, 749-760.

Malek MH, Olfert IM \& Esposito F (2010). Detraining losses of skeletal muscle capillarization are associated with vascular endothelial growth factor protein expression in rats. Exp Physiol 95, 359-368.

Mrazkova O, Grim M \& Carlson BM (1986). Enzymatic heterogeneity of the capillary bed of rat skeletal muscles. Am J Anat 177, 141-148.

Nagase H \& Woessner JF, Jr. (1999). Matrix metalloproteinases. J Biol Chem 274, 21491 21494.

Olfert IM \& Birot O (2011). Importance of Anti-angiogenic Factors in the Regulation of Skeletal Muscle Angiogenesis. Microcirculation 18, 316-330.

Olfert IM, Breen EC, Gavin TP \& Wagner PD (2006). Temporal thrombospondin-1 mRNA response in skeletal msucle exposed to acute and chronic exercise. Growth Factors 24, 253-259.

Olfert IM, Breen EC, Mathieu-Costello O \& Wagner PD (2001). Skeletal muscle capillarity and angiogenic mRNA levels after exercise training in normoxia and chronic hypoxia. J. Appl. Physiol. 91, 1176-1184.

Olfert IM, Howlett RA, Tang K, Dalton ND, Gu Y, Peterson KL, Wagner PD \& Breen EC (2009). Muscle-specific VEGF deficiency greatly reduces exercise endurance in mice. J Physiol 578, $1755-1767$.

Olfert IM, Howlett RA, Wagner PD \& Breen EC (2010). Myocyte vascular endothelial growth factor is required for exercise-induced skeletal muscle angiogenesis. Am J Physiol Regul Integr Comp Physiol 299, R1059-1067.

Prior BM, Yang HT \& Terjung RL (2004). What makes vessels grow with exercise training? $J$ Appl Physiol 97, 1119-1128.

Richardson RS, Wagner H, Mudaliar SR, Henry R, Noyszewski EA \& Wagner PD (1999). Human VEGF gene expression in skeletal muscle: effect of acute normoxic and hypoxic exercise. Am J Physiol 277, H2247-2252.

Roudier E, Gineste C, Wazna A, Dehghan K, Desplanches D \& Birot O (2010). Angioadaptation in unloaded skeletal muscle: new insights into an early and muscle typespecific dynamic process. J Physiol 588, 4579-4591. 
Rullman E, Norrbom J, Stromberg A, Wagsater D, Rundqvist H, Haas T \& Gustafsson T (2009). Endurance exercise activates matrix metalloproteinases in human skeletal muscle. $J$ Appl Physiol 106, 804-812.

Shi H, Huang Y, Zhou H, Song X, Yuan S, Fu Y \& Luo Y (2007). Nucleolin is a receptor that mediates antiangiogenic and antitumor activity of endostatin. Blood 110, 2899-2906.

Slater M \& Murphy CR (1999). Thrombospondin is sequentially expressed and then deexpressed during early pregnancy in the rat uterus. Histochem $J \mathbf{3 1}, 471-475$.

Srivastava M \& Pollard HB (1999). Molecular dissection of nucleolin's role in growth and cell proliferation: new insights. Faseb J 13, 1911-1922.

Tang K, Breen EC, Gerber HP, Ferrara NM \& Wagner PD (2004). Capillary regression in vascular endothelial growth factor-deficient skeletal muscle. Physiol Genomics 18, 6369.

Volpert OV, Pili R, Sikder HA, Nelius T, Zaichuk T, Morris C, Shiflett CB, Devlin MK, Conant K \& Alani RM (2002). Id1 regulates angiogenesis through transcriptional repression of thrombospondin-1. Cancer Cell 2, 473-483.

Yamaguchi N, Anand-Apte B, Lee M, Sasaki T, Fukai N, Shapiro R, Que I, Lowik C, Timpl R \& Olsen BR (1999). Endostatin inhibits VEGF-induced endothelial cell migration and tumor growth independently of zinc binding. Embo J 18, 4414-4423. 


\section{FIGURE LEGEND}

Figure 1. Maximal running test showing aerobic exercise capacity prior to training (pretraining), post-training and after detraining. Measured using a standardized incremental maximal exercise protocol on a rodent treadmill. ${ }^{*} p<0.05$ compared to pre-training. Data are mean \pm SE.

Figure 2. Basal protein expression of vascular endothelia growth factor (VEGF), thrombospondin-1 (TSP-1; 165kDa), matrix metalloproteinase (MMP)-2, MMP-9, endostatin $(22 \mathrm{kDa})$ and nucleolin $(100 \mathrm{kDa})$ in the (a) gastrocnemius, (b) plantaris and (c) soleus muscles measured by ELISA or Western Blot. Representative blots are shown. Control mice did not train, all others voluntarily trained on running wheels for 21 days and then subsequently detrained for $1,7,14$, or 28 days (D1, D7, D14, D28, respectively). a.u.= arbitary densitometry units from Western blot analysis for each respective factor which has been normalized to the housekeeping gene GAPDH or $\beta$-tubulin. ${ }^{*} p<0.05$ compared to control. Data are mean \pm SE.

Figure 3. Fold-change in protein expression in response to acute exercise for VEGF, endostatin $(22 \mathrm{kDa})$ and nucleolin $(100 \mathrm{kDa})$ in the gastrocnemius, plantaris, and soleus following the same conditions in Figure 2. These proteins (VEGF, endostatin and nucleolin ) were selected due to their increased expression 4 hours post acute exercise in the Training Study (Chapter 2). The basal protein expression in each group of mice was set to 1.0 showing the relative acute/basal protein change by group. Data reflect protein levels 4 hours post acute exercise. ${ }^{*} p<0.05$ to basal within group. Data are mean \pm SE. 


\section{$\underline{\text { TABLES AND FIGURES }}$}

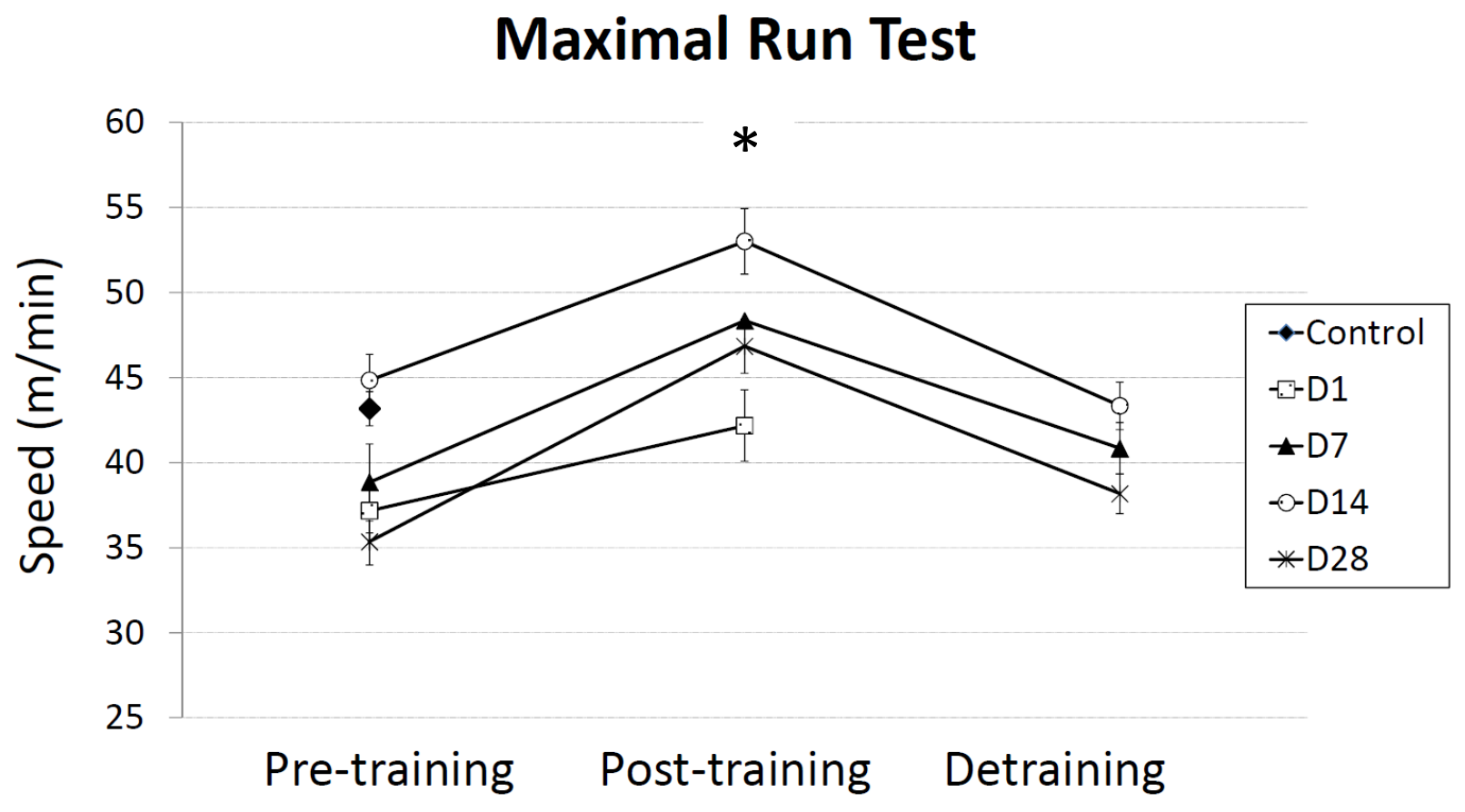

Figure 1. 

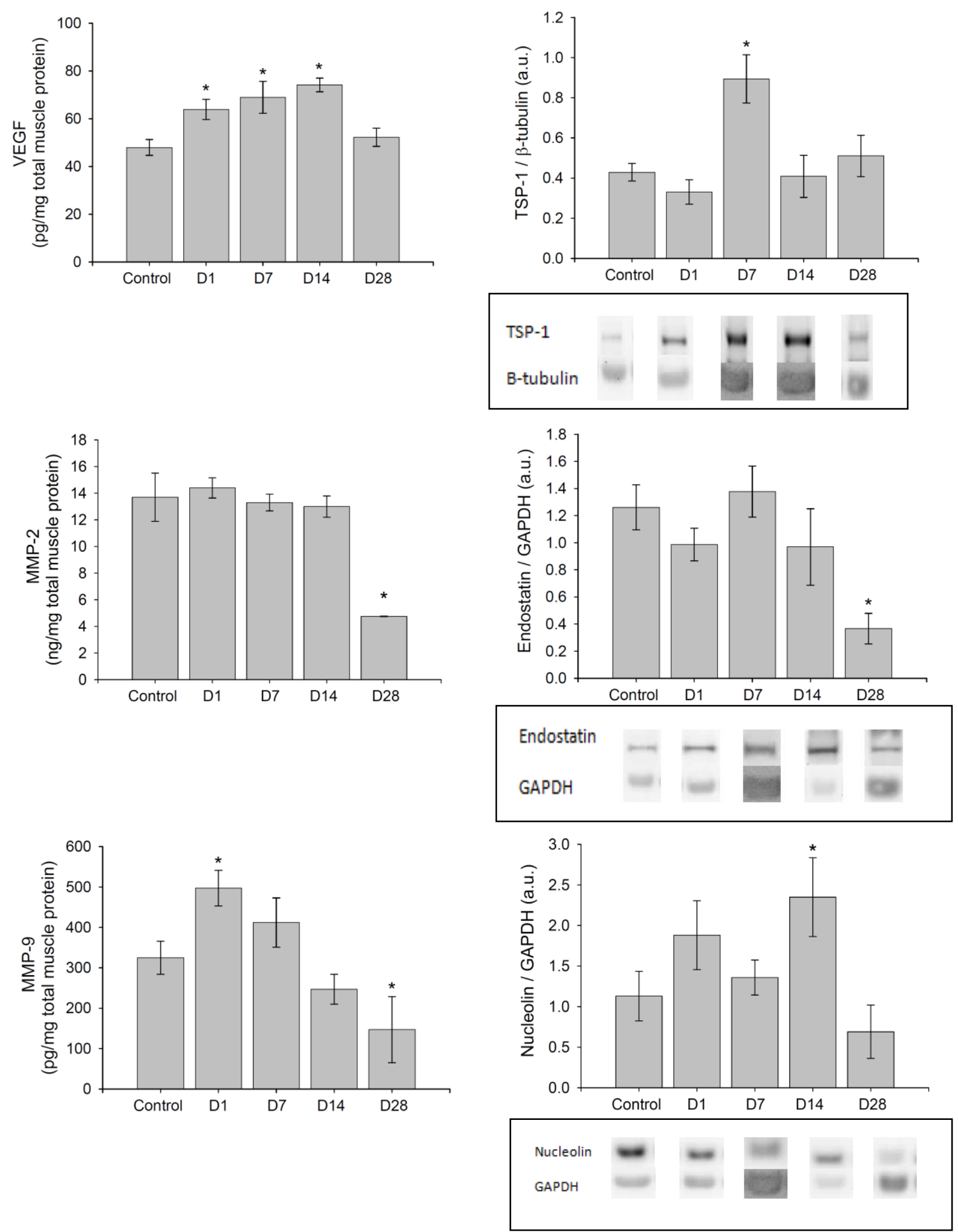

Figure 2a. (Gastrocnemius) 

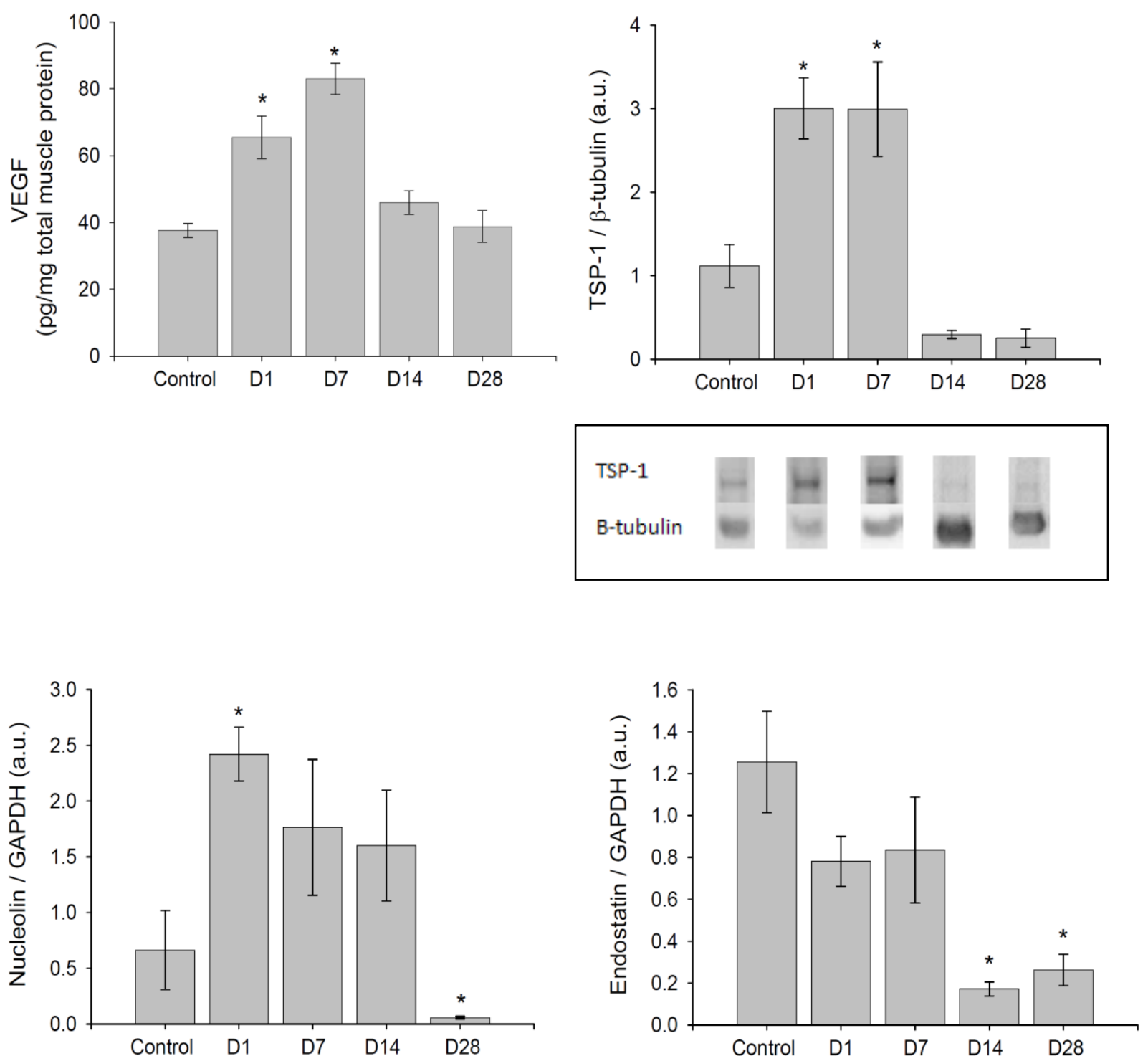

Nucleolin

GAPDH

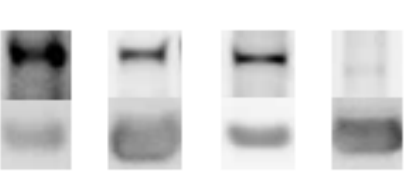

Endostatin

GAPDH

Figure 2b. (Plantaris) 

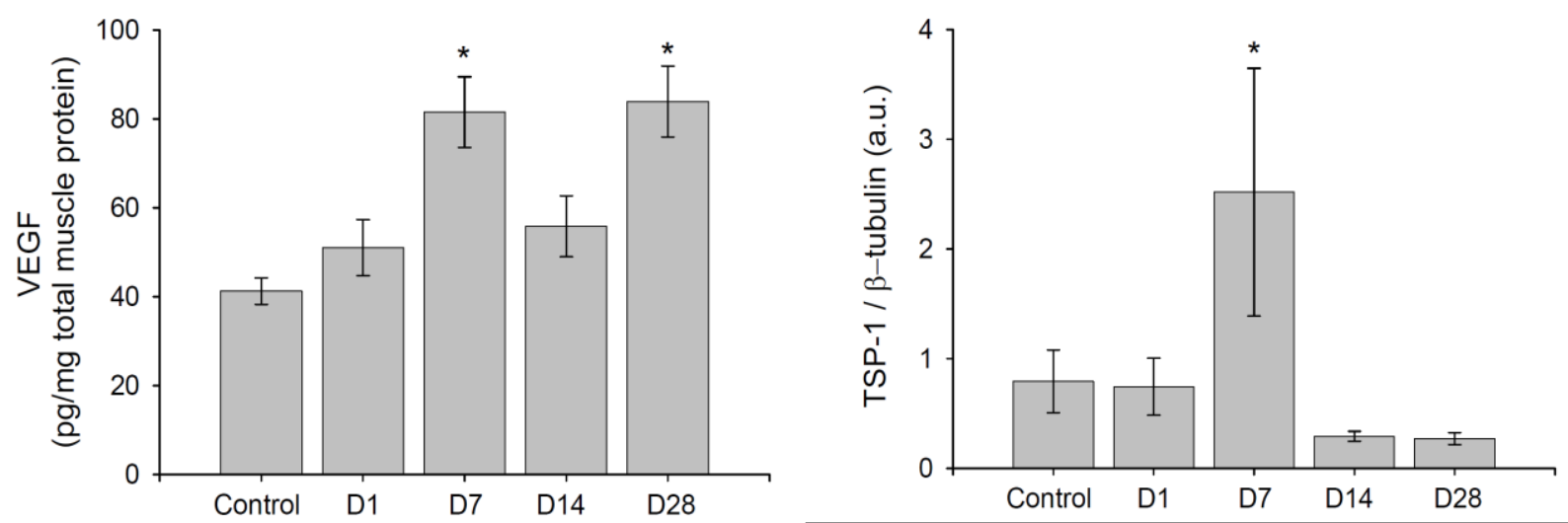

TSP-1

B-tubulin
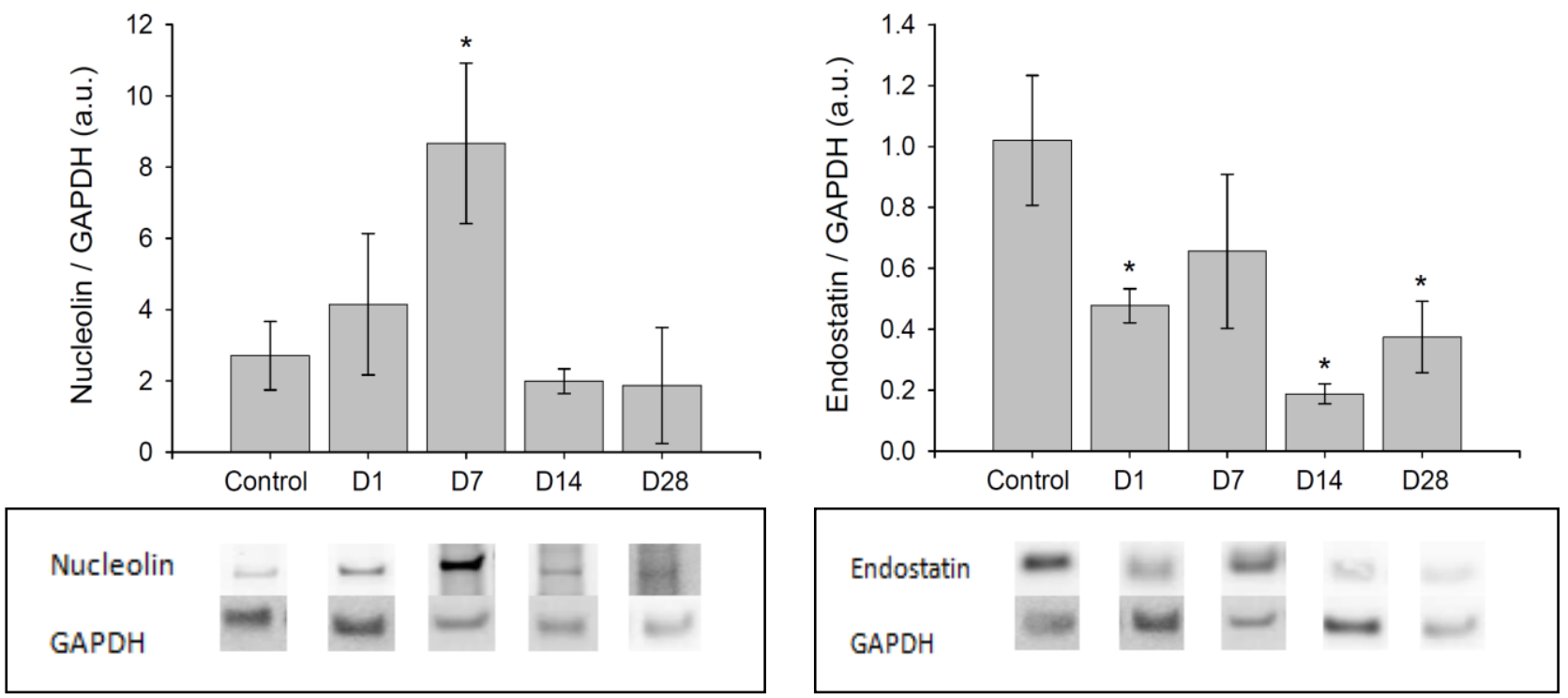

Figure 2c. (Soleus) 

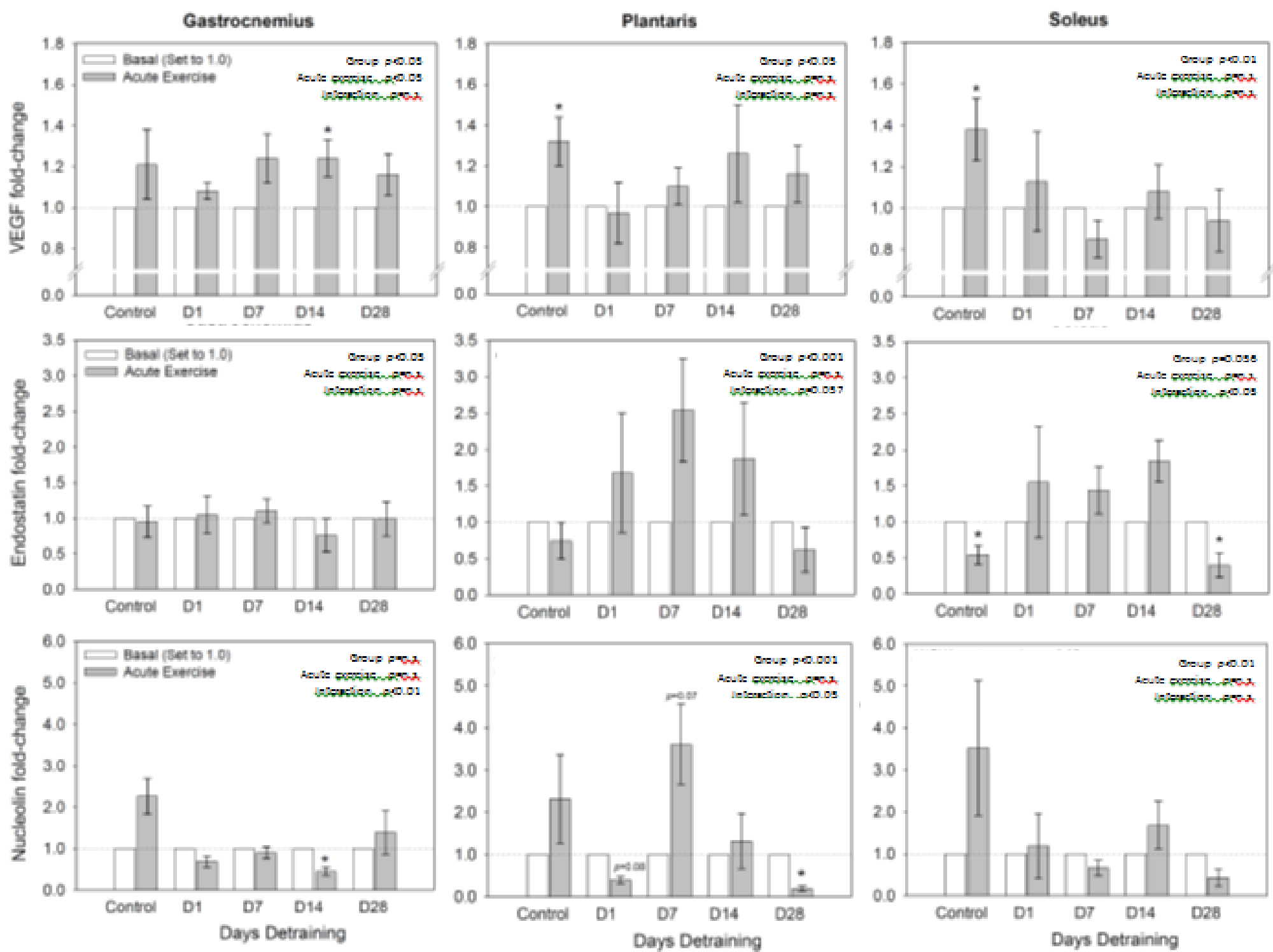

Figure 3. 


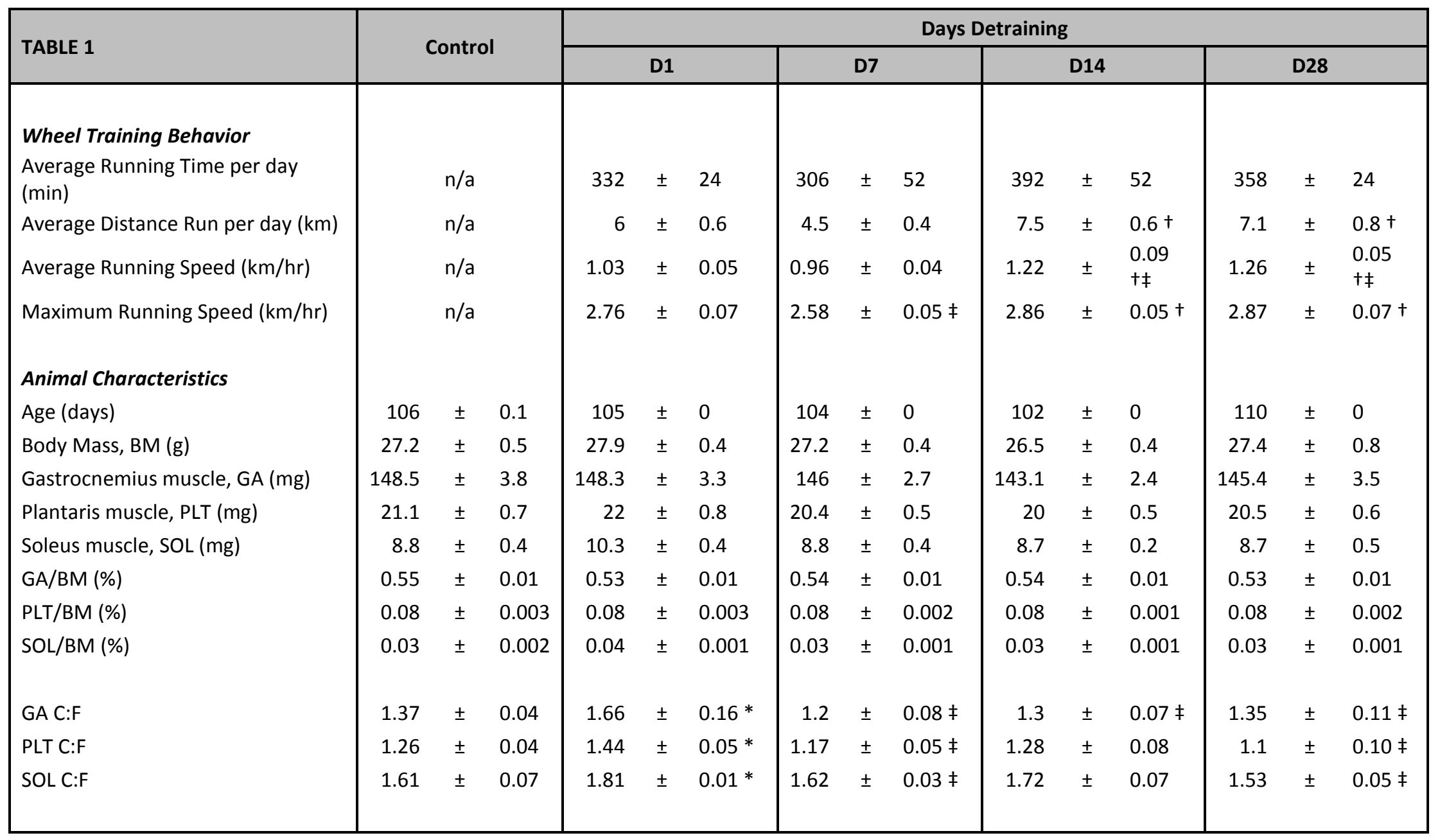

Mean \pm SEM. n/a, not applicable; C:F, capillary-to-fiber ratio. $p<0.05$; ₹ different compared to control; ${ }^{*}$ different compared to D1; + different compared to $D 7$.

Table 1. Characterization of wheel running over 21 days, age, body mass, muscle mass and C:F ratio. 


\section{CHAPTER 4: CONCLUSION}

\section{Conclusions}

This work evaluated the temporal response of several positive and negative angiogenic proteins in response to acute exercise, training and detraining in skeletal muscle of mice. We find and report a complex interplay between positive and negative angiogenic factors in response to these physiologic conditions resulting in capillary growth (training) and regression (detraining).

\section{Temporal Response to Acute Exercise}

We observed that acute exercise elicited a response of VEGF, MMP-2, TSP-1, endostatin and nucleolin proteins, but no significant response was reported for MMP-9 up to 24 hours post exercise. These results were in agreement with our initial hypothesis that both positive and negative angiogenic factors would be up-regulated. We find that angioregulatory proteins were markedly up-regulated in either an early (2-4 hours; VEGF, endostatin, nucleolin) or late (12-24 hours; MMP-2 and a lesser extent TSP-1) phase in the 24 hour time course. We are the first to show that nucleolin, a nuclear protein that may assist in the intracellular transport of VEGF and/or endostatin, may also be responsive to acute exercise. The role of nucleolin in exercise is novel and could provide a link between VEGF and endostatin regulation in response to acute exercise. In both, our Training (Chapter 2) and Detraining Studies (Chapter 3) we observed a consistent and robust VEGF protein response to acute exercise in untrained mice. Surprisingly, the response of both endostatin and nucleolin were much more variable in our Training (Chapter 2) and Detraining Studies (Chapter 3). In the Training Study (Chapter 2), although both proteins were upregulated in the acute exercise time course, neither endostatin nor nucleolin responded to acute exercise in the control mice for the training portion of the study. However, in the Detraining Study (Chapter 3), we find evidence that nucleolin responded 
to acute exercise, but endostatin still did not respond. Perhaps these proteins are more sensitive to the relative exercise workload for each mouse. Given the variability in the responses for both nucleolin and endostatin, the overall importance of these factors may still be somewhat speculative.

We expected a more robust TSP-1 protein response to acute exercise based on its known mRNA response, but TSP-1 tended to only be elevated late (up to 24 hours) post exercise. Likewise, we saw no response for MMP-9, which also was surprising since other studies have reported MMP-9 to increase within a few hours following acute exercise in different animal models. Discrepancies between our data and others may be in part explained by exercise intensity and species type. Nonetheless, we find clear evidence supporting our hypothesis that both positive and negative angiogenic factors are up-regulated in response to acute exercise, suggesting an internal system designed to limit and/or prevent unnecessary utilization of energy and biological resources for capillary expansion in response to a single, one-time, stimulus.

\section{Temporal Response to Training}

The goal of our Training Study was to temporally evaluate and link changes in angiogenic regulators with changes to training induced capillary adaptation. We found that voluntary wheel running increased muscle capillarity after at 7 days. In agreement with our hypothesis, basal expression of positive factors remained elevated while basal expression of negative factors decreased with increasing temporal exposure to training. In response to our hypothesis, nucleolin and VEGF were significantly higher at 5 and 7 days respectively while TSP-1 was diminished at days 5 and 7 , relative to days 14 and 28 of training; these two time points correlate with a significant increase in skeletal muscle capillarity at day 7 . Contrary to our hypothesis, positive factors did not remain elevated, nor did negative factors decrease with 
increasing training; by day 28 VEGF was significantly lower and TSP-1 was significantly higher than it was at days 5 or 7 . Retrospectively, this inverse of the angiogenic balance is feasible because once the skeletal muscle has adapted to the increased demand for oxygen created during exercise, there could likely be negative feedback to inhibit/reduce the continual growth of vasculature. Other angiogenic factors investigated in response to training were MMP-9, MMP-2 and endostatin. MMP-9 and MMP-2 were down (relative to control) and up (relative to day 3) respectively at days 5, 7 and 14; however, previous literature would suggest that MMP-9 should have been up-regulated at day 3 . This discrepancy might be accounted for by exercise intensity and animal models used. Endostatin was not significantly altered with training.

Furthermore, we hypothesized that acute exercise would result in a blunting or loss of the positive, but not negative, angiogenic regulator response following exercise training. In response to this hypothesis, the acute response to VEGF was present at day 3 and remained elevated after 5 days of training. Given the response for endostatin and nucleolin in our previous acute temporal exercise response, we were surprised that neither factor was increased in the control group of the training study. At present, the reason for this remains unclear. Given that muscles were sampled in early phase (i.e 4 hours) post exercise, we would not expect TSP-1 or the MMP-2 to respond.

Taken together, it can be concluded that angiognesis resulting from exercise training occurs in association with basal changes of both positive (VEGF, MMP-2, nucleolin) and negative (TSP-1) angiogenic regulators. Given the differences observed in these proteins between humans and mice, these data also highlight the temporal responses we observed in mice (particularly for VEGF, MMP-2 and MMP-9) may not be directly comparable to humans. Nonetheless, it is clear our results show that there is a complex coordination in the protein responses of both positive and negative angiogenic factors that correspond with traininginduced muscle capillary adaptation. 


\section{Temporal Response to Detraining}

The goal of the Detraining Study was to temporally link changes in angiogenic regulators with capillary regression associated with physical deconditioning (i.e. detraining). We hypothesized there would be in increase of negative, relative to positive angiogenic factors with detraining. In agreement with our hypothesis, we found TSP-1 protein was increased at 7 days post training in all the skeletal muscle examined. At this same time point, skeletal muscle capillarity returned to baseline (pre-training levels) despite elevated VEGF protein. We also hypothesized that the acute response to exercise would return once capillarity reached pretraining levels. To our surprise, this was not the case since 28 days of detraining was not sufficient time for the well-established VEGF response to acute exercise to reappear. Finally, we hypothesized that oxidative muscles would have greater changes in angiogenic regulators (both positive and negative) compared to glycolytic muscles. We show there are protein expression differences between glycolytic and oxidative muscles after training (D1); TSP-1, VEGF and nucleolin were all elevated after 1 day of detraining relative to the control group in the plantaris but not the soleus. However, all muscles (GA, PLT, SOL) had the same increase of both TSP-1 and VEGF protein at 7 days post training (D7), the same time point as capillary regression. We also show a trend for a decrease of endostatin with time spent detraining in all muscles. In conclusion, these data suggest that although detraining responses after 1 day of detraining may be different among the oxidative and glycolytic muscles, all muscles exhibited physiologic regression of skeletal muscle capillary regression by 7 days of detraining which may be due to a greater influence of negative regulators (TSP-1) than positive regulators (VEGF) following detraining. Furthermore, we have shown that the VEGF protein response to acute exercise remained blunted after 28 days of detraining.

In conclusion, our findings suggest that skeletal muscle angiogenesis is a complex interplay involving both positive and negative angiogenic factors whose expression profiles are 
temporally distinct in response to acute exercise. And, whose relative importance may be different in response to training-induced capillary expansion compared to detraining-induced capillary regression. Future temporal studies investigating additional angiogenic factors may assist in clarifying the mechanisms underlying changes in skeletal muscle capillarity which will aid in promoting our understanding of capillary growth and regression under physiologic conditions, but also lay the foundation to better understand the reasons for capillary rarefaction in chronic disease such as chronic obstruction pulmonary disease, diabetes and/or heart disease. 